\title{
Guerre et récit chez les Indiens ayorés du Chaco boréal paraguayen
}

Salvatore D'Onofrio

\section{OpenEdition}

1 Journals

Édition électronique

URL : https://journals.openedition.org/jsa/3743

DOI : 10.4000/jsa.3743

ISSN : 1957-7842

Éditeur

Société des américanistes

Édition imprimée

Date de publication : 1 janvier 2003

Pagination : 39-81

ISSN : 0037-9174

\section{Référence électronique}

Salvatore D'Onofrio, «Guerre et récit chez les Indiens ayorés du Chaco boréal paraguayen », Journal de la Société des américanistes [En ligne], 89-1 | 2003, mis en ligne le 16 janvier 2008, consulté le 04 septembre 2022. URL : http://journals.openedition.org/jsa/3743 ; DOI : https://doi.org/10.4000/jsa. 3743 


\title{
GUERRE ET RÉCIT CHEZ LES INDIENS AYORÉS DU CHACO PARAGUAYEN
}

\author{
Salvatore D'ONOFRIO *
}

Chez les Indiens ayorés du Chaco paraguayen, la guerre n'est pas seulement pratiquée, elle est aussi racontée. Le droit de raconter ne revient en principe qu'à ceux qui ont déjà tué. Il s'agit d'une pantomime peu répandue chez d'autres Indiens des basses terres et qui s'apparente au mythe en raison de la manière canonique dont elle se déroule et des connexions qu'elle établit avec d'autres aspects de la culture ayoré : la peinture corporelle, la chasse, les pouvoirs chamaniques, les activités rituelles. Forcée à s'inscrire dans l'horizon symbolique pour qu'elle soit efficace, la guerre ayoré se modèle selon la logique de la prédation telle qu'elle a été mise en valeur dans plusieurs travaux récents mais en confirmant, surtout par l'analyse des substances et des gestes, sa possible conversion en relation d'échange. [MOTS cLÉs : guerre, récit, pantomime, Indiens ayorés, Chaco paraguayen, ornement, peinture faciale, activité rituelle, échange.]

Warfare and Story among the Ayoreo Indians of the Paraguayan Chaco. For the Ayoreo Indians of the Paraguayan Chaco, warfare is not something just to be fought, it is also something to be recounted in stories. The right to tell the story belongs in principle only to those who have already killed. This sort of pantomime, little known among other Indians of the lowlands, is related to myth by virtue of the canonical manner in which it unfolds and of the connexions it establishes with other aspects of Ayoreo culture : body painting, hunting, shamanic powers, ritual activities. Ayoreo warfare-compelled in order to be effective to inscribe itself within a symbolic horizon - is modelled on the basis of the logic of predation, as has been emphasized in several recent works, but confirms - above all through the analysis of substances and gestures - its possible conversion into an exchange relationship. [KEY wORDS : warfare, story, pantomime, Ayoreo Indians, Paraguayan Chaco, ornament, body painting, ritual activities, exchange.]

La guerra y sus relatos entre los ayoreo del Chaco paraguayo. Entre los indigenas ayoreo del Chaco paraguayo, la guerra no es solamente practicada sino también relatada. El derecho de contar está reservado en principio a los que ya han matado. Se trata de una pantomima poco difundida entre las otras etnias de las tierras bajas y que se aparenta con el mito por la forma canónica en la que se desarrolla y también por la conexión que

* Université dePalerme (Italie)et Laboratoire d'anthropologie sociale, Paris [s.donofrio@libero.it]. Journal de la Sociétédes Américanistes, 2003, 89-1 : p. 39 à 81 . Copyright OSociété des Américanistes. 
establece con otros aspectos de la cultura ayoreo : la pintura corporal, la caza, los poderes chamánicos, las actividades rituales. Obligada a inscribirse dentro del universo simbólico para ser eficaz, la guerra ayoreo se modela conforme a la lógica predatoria como ha sido discutido en varios trabajos recientes, pero manifiesta a la vez, sobre todo cuando se analizan las sustancias y los gestos, su posible conversión en una relación de intercambio. [PALABRAS ClAVES : guerra, relato, pantomima, indígenas ayoreo, Chaco paraguayo, adorno, pintura corporal, actividad ritual, intercambio.]

La nuit tombe sur le campement [...]. Chaque homme a planté sa lance près de lui ; assis, il s'y appuie parfois; tous fument, tirant lentement de longues bouffées de leurs pipes, pièces rectilignes d'un bois dur, creusé dans le sens de la longueur. Les conversations vont leur train : elles s'animent et se colorent lorsqu'un des participants raconte certains événements d'importance dont il a été témoin ; le conteur se lève alors et imite le comportement des divers protagonistes du drame, qu'il s'agisse d'êtres humains ou d'animaux; il saisit parfois sa lance ou son arc, reproduisant le geste du chasseur qui essaye de tuer sa proie, ou encore il rampe sur le sol, comme un serpent vers sa victime. Il joue une véritable pièce de théâtre et le public participe activement au spectacle : éclats de rire, exclamations, cris d'effroi, soupirs, encouragements accompagnent les paroles et les gestes de l'acteur.

Lucien Sebag (1965).

« La guerre, je vous dis la guerre ». Cette expression est chère aux linguistes, car elle illustre un problème dont la solution est au centre des innovations introduites par Ferdinand de Saussure dans son Cours de linguistique générale ${ }^{1}$. Il s'agit du contenu psychologique différent communiqué par la répétition d'un mot dans la même phrase, mais aussi de la possibilité qu'en dépit des multiples valeurs ou réalisations phoniques d'un mot, on puisse toujours remonter à quelque chose qui leur est commun, quelque chose qui est exprimé par la langue et qui assure la communication entre les hommes. En effet, s'il est vrai que le mot « guerre » évoque à la fois les fanfares et les défilés glorieux des troupes, un frère mort ou une maison détruite, s'il est vrai que von Clausewitz fera référence au prolongement de la politique par d'autres moyens alors que le soldat Schweik pensera à des mots qu'on ne peut pas prononcer pour garder le décor, il est aussi vrai que ces différents sens de " guerre » peuvent coexister dans le savoir des locuteurs car il y a bien quelque chose qui demeure identique. 
Les anthropologues aussi reconnaissent l'unité de l'homme à travers sesmultiples réalisations, quoique certains nient cette possibilité ou bien raccourcissent le trajet afin de dépasser rapidement une variété qui apparaît fastidieuse.

Mais la phrase de Ferdinand de Saussure, citée au début de cet article, a également un sens que la langue française révèle mieux que d'autres langues romanes et qui nous offre la possibilité de réfléchir sur la guerre sous un angle tout à fait particulier. Dire la guerre c'est aussi la raconter et les formes du récit ne sont pas détachées de la pratique ni de l'organisation des sociétés que nous étudions.

Nous avons exploré cette relation entre le récit et la guerre chez les Indiens ayorés ${ }^{2}$, dernier groupe du Chaco boréal à avoir subi une acculturation par les Blancs. Les Ayorés comptent actuellement environ 4500 personnes, dont 2500 vivent en Bolivie (depuis les années quarante) et 2000 au Paraguay (à partir des années soixante). Les Ayorés du Paraguay, chez lesquels nous avons mené notre enquête, se répartissent entre le haut Paraguay (villages de Cucaani, Isla Alta, Guidai Icciai) et le Chaco central (villages de Campo Loro, Ebetogué, Jesudi, Aocójnandí, Túnucojnai). Un petit groupe vit en permanence dans la banlieue de la ville mennonite de Filadelfia, alors qu'un autre groupe d'une centaine de personnes n'a jamais eu de contacts avec la « civilisation des Blancs ». Les Ayorés ne sont plus éparpillés sur un territoire continu, comme l'iñdiquent encore quelques cartes de la région (Figure 1) qui montrent néanmoins l'étendue de l'espace où ils pratiquaient, avant leur sédentarisation intégrale, les activités de chasse, de récolte et de jardinage. Il n'empêche que le processus d'acculturation n'a pas entamé quelques traits significatifs de la vie sociale ayoré comme l'organisation clanique, la forme circulaire des villages, la famille élargie. La résidence est uxorilocale, le mariage est interdit entre cousins (que l'on considère comme des germains) et entre individus du même clan ${ }^{3}$. Des petits morceaux de terre autour des villages permettent aux Ayorés de continuer à pratiquer leurs cultures traditionnelles ${ }^{4}$ auxquelles ils intègrent aujourd'hui des arbres fruitiers et le manioc, un peu d'élevage et, dans les villages du haut Paraguay, la récolte d'appâts destinés aux pêcheurs qui arrivent de Puerto Murtiño, petit port brésilien de l'autre côté du fleuve. Des récoltes et des expéditions de chasse, souvent collectives, sont organisées, mais l'activité la plus rentable pour les Ayorés est le travail comme salariés dans les fermes des Mennonites dans le Chaco central. Des objets traditionnels en bois, des plumes tressées, des armes et des sacs de fibre végétale sont devenus des produits artisanaux très recherchés au Paraguay.

\section{LE RÉCIT}

Chez les Indiens ayorés du Chaco boréal, la position centrale du récit tient non seulement au fait qu'il permet de socialiser les événements et d'en légitimer les effets, mais aussi à la manière même dont il se déroule. Au retour des 


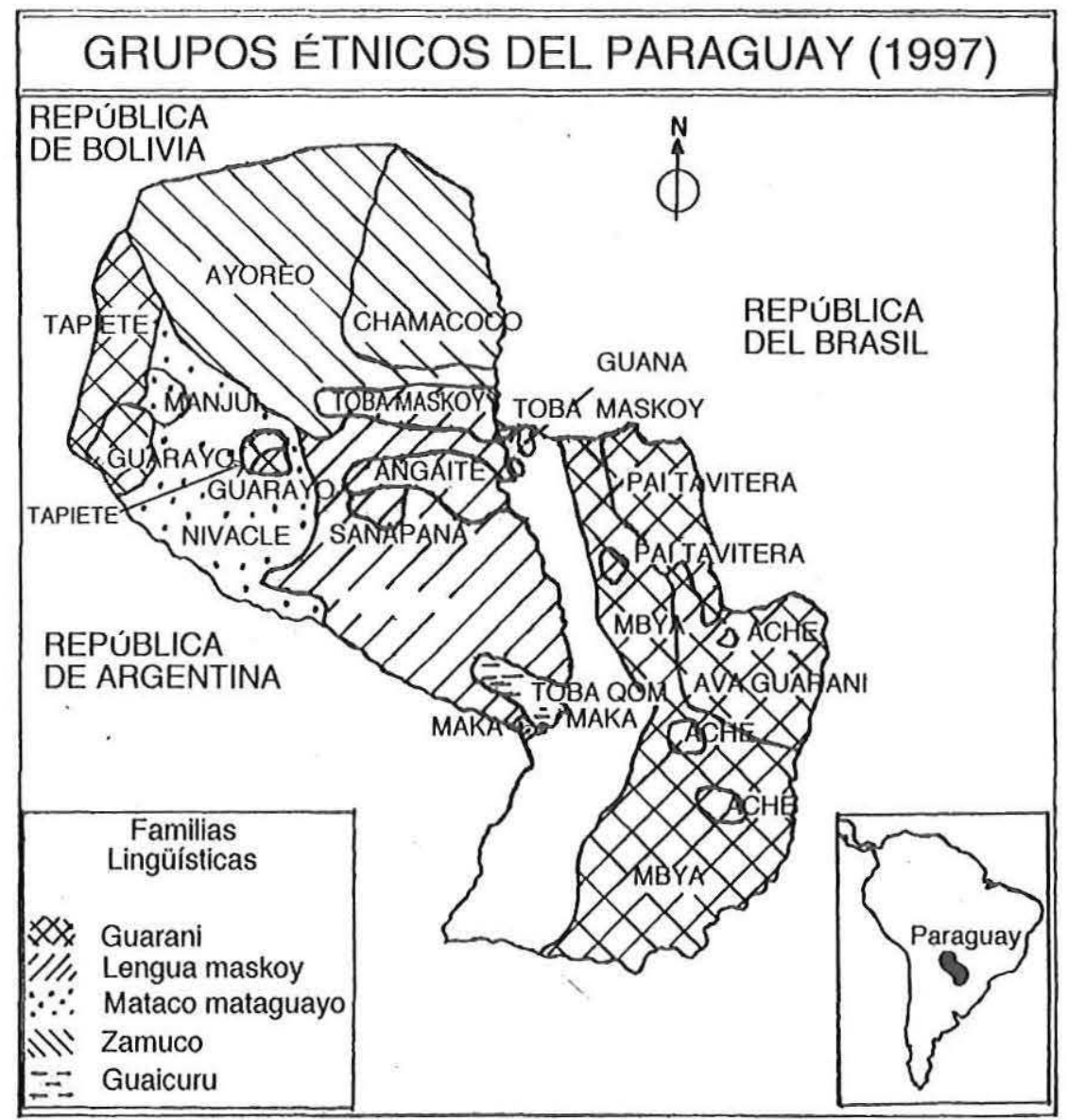

FIG. 1. - Carte des groupes ethniques du Paraguay (Bartolomé 2000)

expéditions guerrières, ceux qui y ont participé attendent quelques jours avant de raconter les faits et ce droit ne revient en principe qu'à ceux qui ont tué. Il est inséparable d'une obligation fondamentale de la culture ayoré : raconter les mythes ou, au moins, y faire référence dans toutes les occasions qui le réclament, d'après une conception qui veut que les animaux, les choses et jusqu'à des entités abstraites, se soient formés in illo tempore par métamorphose d'une forme humaine originelle (jnani bajai, pl. jnani bajade, ancêtres) que seuls les Ayorés actuels ont gardée. "Quand le vautour, le charbon ou le courage étaient encore des Ayorés [...]", c'est-à-dire des personnes, selon l'incipit des mythes qui les 
concernent. Raconter les mythes revient en quelque sorte à revivre le passage qui a conduit à la séparation actuelle entre les Ayorés et le reste de l'univers, tout en inscrivant chacun de ses éléments constitutifs dans l'un des sept clans auxquels les Ayorés eux-mêmes appartiennent. Cette conception grandiose, que nos collègues argentins ont interprétée suivant des postulats phénoménologiques de type éliadien et que Lucien Sebag (1965) et Carmen Bernand (1977) ont lue à la lumière de l'anthropologie structurale, est la toile de fond de tous les aspects de la vie sociale ayoré, y compris la guerre, ainsi que les tabous alimentaires ou les relations entre les sexes. Les récits ayorés de guerre ont encore une caractéristique qui les apparente aux mythes, à savoir une formalisation canonique très stricte qui se manifeste essentiellement de deux manières : par la répétition presque identique du récit tel qu'il a été proposé la première fois et par une articulation également identique du discours indépendamment du groupe (territorial ou clanique) auquel le guerrier ayoré appartient ou du style qui lui est propre ${ }^{5}$.

Nous prenons le déroulement canonique de cette forme narrative comme point de départ d'une analyse visant à explorer, d'une part la pratique de la guerre dans ses différentes phases - visions chamaniques, préparation corporelle, techniques de combat, purifications -, d'autre part les conséquences qu'elle entraîne et l'idéologie qui la soutient : définition des pouvoirs, relations intratribales, rapports entre les genres.

On a affaire à une pantomime peu répandue chez les Indiens des basses terres, par laquelle les Ayorés racontent leurs expéditions guerrières ainsi que leurs rencontres périlleuses avec les jaguars. L'espace du récit est généralement le campement, qu'il s'agisse du campement permanent (guidai en ayoré), qui est occupé durant 4 à 6 mois à la saison des pluies et des plantations, ou des campements temporaires (deguode, sing. degui) de saison sèche, souvent réduits aux feux nocturnes. Pendant cette saison, de 6 à 8 mois, les Ayorés ne vivent que de chasse et de cueillette, auxquelles s'ajoutent quelques maigres réserves alimentaires de la saison précédente (surtout des haricots et du maïs). Chacun raconte devant son habitation, entouré en principe par les membres de son jogasui, la famille élargie qui est encore aujourd'hui le lieu véritable de socialisation ainsi que de définition des pouvoirs et des échanges en tout genre avec les autres groupes. Cette forme narrative ne reçoit pas d'autre nom que celui de "récit » (gojñai) à la différence des mythes, surtout d'origine, qui sont qualifiés et racontés de façon spécifique (Bormida 1973). À un récit de guerre, pocaningai en ayoré, le conteur peut en ajouter d'autres, transformant ainsi cette occasion en une séance narrative de longue durée dans laquelle d'autres membres du jogasui peuvent également intervenir avec leurs exploits.

Le guerrier-conteur (qui est aussi un horticulteur et un chasseur-cueilleur car la guerre, bien que décidée et organisée par les dacasutiede, les chefs, n'est pas une activité spécialisée chez les Ayorés), s'exhibe le plus souvent quelques jours après son retour au campement. Mais, à partir de ce moment, le récit entre dans son 
répertoire personnel que tout le monde connaît et qui pourra être exécuté spontanément ou bien sur sollicitation d'autrui. Il peut arriver alors que son récit soit interrompu, anticipé ou souligné par un public toujours attentif dont la participation n'est jamais repoussée, ce qui n'empêche cependant pas le conteur d'organiser l'espace du récit de manière indépendante, car l'effort fait pour se replonger dans l'événement meurtrier peut même l'amener, dans sa mise en scène, à tourner le dos à ses auditeurs. L'espace de la narration est en tout cas presque toujours l'objet d'attentions particulières, qu'il s'agisse de le délimiter par le geste mimant un jaguar en train de sauter ou bien de faire mine de le nettoyer avec les pieds ou le manche des armes utilisées pour la représentation ${ }^{6}$.

Ce cadre chronotopique est complété par un traitement du corps qui contribue à qualifier le récit de lieu de ré-immersion périodique dans la situation de guerre et, à travers elle, dans le temps des origines également. Avant son récit, le conteur peint et orne son corps de la même manière que pour l'expédition guerrière. Ce sont des signes qui lui donnent symboliquement la puissance des animaux dont il utilise la peau et les plumes : l'ayoi, bandeau frontal qui combine ces deux matériaux prélevés dans la nature ; le cobidié, collier de plumes d'oiseaux divers, que l'on met autour du cou, la partie concave vers le haut ; les potadié, plumes d'oiseaux divers, surtout plumes de perroquets tressées qui tombent sur le dos ; parfois des bracelets, maniopiede, et souvent aussi des ceintures auxquelles on accroche les carapaces de petites tortues qui sont des meurtrières dans le mythe et dont le nombre sur le corps du guerrier-conteur indique le nombre de personnes qu'il a tuées ${ }^{7}$. En revanche, on ne met pas au cou les sifflets (potá) de bois de palo santo que l'on fait résonner après avoir tué ou bien lors de la fête d'Asoonjá, l'engoulevent mythique des Ayorés dont le chant, vers la mi-août ${ }^{8}$, est censé marquer la fin de la saison sèche et le début de la saison des pluies (même si, en réalité, celle-ci peut tarder encore deux ou trois mois). Signalons deux éléments importants : premièrement, seul le guerrier qui a déjà tué a progressivement droit à ces ornements. Leur acquisition constitue en quelque sorte un rite initiatique, certes dilué dans le temps et dans une société où, à proprement parler, il n'y en a pas. Les plus jeunes peuvent participer aux expéditions guerrières et se peindre, ils n'ont alors droit qu'à quelques ornements, par exemple des petites plumes blanches collées sur la poitrine avec de la cire d'abeille pour signaler qu'« ils pensent déjà à la guerre » ou un bandeau frontal (ayoi dajua) en fibre de cáaguatá, une broméliacée, et certainement pas en cuir. Pour pouvoir utiliser ce dernier, il faut avoir tué, tandis que certains ornements, comme les grandes plumes du héron (cobia), sont les attributs du chamane seulement. Deuxièmement, la peinture corporelle porte le même nom que le maquillage que les jeunes filles utilisent pour séduire leurs amants dans la période longue, turbulente et joyeuse des amours juvéniles.

La pantomime du récit de guerre prévoit aussi l'usage d'armes qui, souvent, ne sont pas celles qui ont tué car ces dernières sont considérées comme impures et 
généralement abandonnées immédiatement après la tuerie, au cours d'un rituel de purification du sang. Par cette pantomime, on se limite à raconter les gestes de tuerie que l'on peut ordonner en deux catégories : d'une part, la mise à mort des hommes, auxquels - au moins de ce point de vue - on associe les jaguars; d'autre part, la mise à mort d'autres animaux comme le pécari ou le tapir, dont la tuerie est davantage racontée pour faire rire que pour montrer sa valeur. Dans cette catégorie, on range aussi la vache et le chien que, quarante ans plut tôt, les Ayorés ne connaissaient que comme animaux des Blancs, alors qu'ils possédaient des chiens qu'ils avaient volés aux Blancs. Le pillage, en réalité, ne motivait explicitement que l'expédition contre les Blancs. Le cas échéant, on volait aussi les autres Ayorés et les femmes étaient appréciées. Le fait d'avoir tué un animal des Blancs ou de la forêt donnait droit à s'orner avec des plumes d'oiseaux qui avaient une certaine puissance mais qui n'avaient pas été des tueurs lorsqu'ils étaient encore Ayorés.

Une hiérarchie, presque admise par tous les Ayorés, confère plus de valeur à celui qui a tué un Blanc, puis à celui qui a tué un autre Ayoré, enfin à celui qui a abattu un jaguar (catai, animal qui, en espagnol populaire paraguayen, est appelé tigre). Tuer un puma (caté, león pour les Paraguayens) ou d'autres animaux est une chose que "même les femmes pourraient faire ». Cela devient néanmoins objet de récit. Contrairement à ceux de Bolivie (Bormida 1979), les Ayorés du Paraguay ne comptabilisent généralement pas, parmi leurs victimes, les animaux des Blancs. Ils ne font pas non plus référence à d'autres groupes indigènes, certainement du fait qu'ils ne se souviennent plus de cette époque où d'autres Indiens vivaient dans la forêt à portée de leurs raids.

Chacun est fier du nombre de personnes et de jaguars qu'il a tués, et ne laisse passer aucune occasion de le rappeler, notamment parce que la tuerie et les qualités de courage et d'habilité qu'elle implique sont les conditions sine qua non pour se mettre en évidence et accéder au rôle de chef. Le courage, en particulier, est non seulement le résultat de la métamorphose d'un homme du temps des origines, comme l'illustre bien le mythe ${ }^{9}$, mais aussi une qualité très appréciée par les femmes et manifestée par un « état du corps » que surtout les chefs doivent atteindre lors de la préparation à une expédition guerrière. C'est par la guerre qu'un Ayoré confirme ou fait évoluer sa position statutaire au sein des différentes sphères qui définissent son identité ${ }^{10}$. Juste un exemple. Dès qu'un homme se marie, le principe de résidence uxorilocale l'oblige à vivre auprès de son beaupère. Il est ici dans une situation de marginalité et de dépendance. C'est seulement par l'intermédiaire de sa femme qu'il pourra adresser la parole à ses beauxparents et à ses belles-sœurs, et vice versa. C'est encore avec le consentement de sa femme qu'il pourra construire sa propre maison loin de celle de son beau-père lorsque ses enfants auront grandi. Ce n'est que la guerre qui peut lui donner la possibilité d'accélérer ce processus d'émancipation et ce n'est pas un hasard si cette conduite de mutisme s'atténue uniquement dans les expéditions guerrières, 
notamment à travers les conseils que le beau-père peut donner à son gendre. Permettant de convertir en puissance la fragilité d'un homme qui se voit obligé de négocier en permanence ses pouvoirs et ses rôles, la guerre et la tuerie se situent au centre non seulement de la vie sociale des Ayorés mais aussi de leur mémoire. Aujourd'hui, dans une situation largement différente, il arrive que les chefs soient nommés parmi ceux dont le père a tué, comme si cette valeur guerrière se transmettait généalogiquement et pouvait être récupérée à tout moment. Le récit fonctionne à la fois comme un lieu de vérité socialement contrôlée et d'affirmation des valeurs et des pouvoirs impliqués.

Mais, avant d'analyser ces récits, en voici quelques-uns. Leur durée varie entre cinq et vingt minutes. Chaque récit commence normalement par un adverbe de temps ou une locution adverbiale dans une phrase tournée au passé. Celle-ci situe le spectateur au milieu d'un événement dont on annonce de manière synthétique le contenu. La construction morphologique de la narration ayoré (dont Briggs en 1973 a fait une première analyse) confirme cette tendance à la ré-immersion symbolique dans l'événement. Les histoires guerrières ou de meurtre dont tel ou tel a été protagoniste sont si marquantes qu'elles semblent donner lieu à un seul récit dont les différents épisodes sont justement introduits par des adverbes assurant à chaque fois le déplacement spatio-temporel. Nous avons choisi trois exemples parmi les plus courts en donnant leur traduction littérale intégrale ${ }^{11}$.

Kuisi, du clan jnúminí, de Cucaani, dans le haut Paraguay, raconte les deux premiers :

Une fois nous avons poursuivi des Ayorés et quand nous avons trouvé leur campement, j'allai avec les autres : « je suis en train d'aller, je suis en train d'aller, je suis en train d'aller ; je vais les rencontrer, je vais les rencontrer, je vais les rencontrer ».

Nous avons continué à les poursuivre en cherchant où étaient ces Ayorés que nous voulions trouver, et je dis comme ça : " nous sommes en train d'aller, nous sommes en train d'aller, nous sommes en train d'aller, nous sommes en train de les chercher, nous sommes en train de les rencontrer, nous sommes en train de les rencontrer $»$.

Arrivés à un certain lieu, nous nous sommes arrêtés et nous avons fait une petite réunion arrivant à la conclusion que nous ne devions pardonner à personne, nous devions tuer tous ceux que nous rencontrerions face à nous. C'était ça la décision du groupe car, eux, ils ont poursuivi et tué quelques-uns de nos proches parents et maintenant ce serait une vengeance si nous les rencontrions.

Quand nous nous sommes approchés de leur campement, un homme était en train de prendre du miel dans un arbre ; c'était un de leurs chefs. Quand il entendit nos cris, il apparut sur le chemin où nous étions en train d'aller et il dit comme ça : " est-ce que c'est vous ? Est-ce que c'est vous », il dit, et nous, nous répondîmes : « c'est nous, c'est nous, et nous allons vous tuer $"$, nous répondîmes.

Ainsi, quand nous arrivions presque au campement nous nous rappelions l'enseignement de mon beau-père qui disait : " si vous attaquez un campement, ne vous inquiétez pas si vous n'êtes pas grands, luttez comme vous pouvez, et ne planifiez pas beaucoup 
car le résultat ne dépend pas de ça "; donc je pensais à ce que mon beau-père disait à ce moment.

Quand j'étais en train d'aller au campement donc, je pensais à ce que mon beau-père m'avait dit, à savoir que n'étant pas des chefs, nous aurions dû faire ce que nous voulions. Mais ce qui était important était ce que le chef ou ceux qui étaient devant nous faisaient car ils étaient ceux qui devaient se mettre en évidence dans un combat.

Quand j'arrivai au campement, je m'arrêtai un moment et je vis que quelques femmes étaient en train de s'échapper et je vis une personne qui ressemblait exactement à une de nos femmes et qui était un peu plus grande qu'elle. Quand je vis cette femme, je levai ma lance et je l'envoyai sur elle.

Je crus ne pas l'avoir frappée mais, tout de suite, je vis que j'avais frappé son bras. La femme tomba par terre et cria : « Eh eh eh » et, par terre, elle parvint à extraire la lance, la jeta à terre et courut ; cette femme à ce moment avait un frère à ses côtés.

Ainsi quand le frère de la femme me vit, il s'approcha avec son bâton de défense, lui vers moi et moi vers lui, et donc nous nous battîmes et je crois qu'il me frappa deux fois avec son bâton et après, quand nous étions en train de nous battre, le bâton de mon ennemi se cassa contre ma lance, mais nous continuions à nous battre l'un contre l'autre avec autant de vigueur.

Nous étions en train de lutter, de lutter, de lutter parce que mon jeune ennemi attrapa ma lance et voulait s'en emparer, je l'attrapai très fort, très fort, mais il voulait toujours me l'arracher, il voulait s'emparer de ma lance pour me tuer, mais je l'empoignais aussi et nous continuions à lutter, à lutter, à lutter.

Et donc quand nous étions en train de combattre, de combattre, de combattre, soudainement vint un de mes compagnons, Jobúi, qui le frappa au côté et après je le coupai avec ma lance lorsqu'il tomba sur le sol.

Je dis à la victime dès qu'il était mort : « nous faisons comme ça avec vous quand nous vous tuons, quand nous gagnons, nous vous cassons comme je suis en train de le faire, en coupant, en coupant, en coupant. Jamais vous ne pourrez nous avoir car nous sommes plus forts que vous ", et donc je continuais à couper, à couper son corps. Je coupai la tête et je la tirai comme ça d'un côté.

Ainsi quand nous tuâmes le jeune, j'attrapai sa tête et je l'emmenai avec moi et nous allâmes où étaient mes camarades : «j'allais, j'allais, j'allais » là où mes camarades étaient.

Ainsi quand mes camarades me virent : « c'est bien comme ça, c'est bien comme ça, c'est ça ce que nous voulons, eux nous ont beaucoup maltraités et c'est comme ça qu'il faut faire avec eux : leur couper la tête, afin que ce soit un exemple aussi pour eux ").

Nous nous réunîmes donc un peu plus loin du lieu où les faits s'étaient passés. Jobui tira une autre tête et la jeta là. Et en dernier Cucciajútodé tira la tête d'une autre femme et la jeta là. Tout le monde les mit ensemble.

Le deuxième récit concerne l'attaque d'une ferme isolée des Mennonites, des colons néo-évangéliques provenant du Canada qui se sont installés dans le Chaco paraguayen depuis les années 1930 suite aux concessions accordées par l'État paraguayen (enseignement en allemand, dispense du service militaire, etc.) : les Mennonites ont été le fer de lance de la pénétration paraguayenne dans les territoires ayorés. Dans la partie initiale du récit, on nomme la peinture 
faciale, ce qui est rare et, sans doute, une adaptation du récit au profit de l'anthropologue :

Quand nous sommes allés chercher les Blancs pour les attaquer, nous sommes allés et nous les avons attendus, en réalité nous étions tout près d'eux, nous sommes restés là et nous les avons regardés.

Quand nous étions tout près des Blancs, nos chefs étaient en train de faire leur peinture autour de nous. Ainsi, ils se sont préparés et ils sont allés : " Ils sont là, nous allons arriver chez eux $"$.

Cucciajútodé, un des chefs qui était très en vue, dit à ce moment : " vous devez faire comme moi pour tuer tous ceux qui sont dans cette maison, parce que j'ai de l'expérience, j'ai déjà tué des Blancs ». Cependant, quelques chefs ont dit qu'ils allaient faire autrement et ils n'accueillirent pas l'idée de Cucciajútodé ni son expérience.

Quand nous sommes arrivés à côté de l'endroit qui était nettoyé, parce que les Blancs nettoyaient une certaine partie autour de la maison où ils vivent, nous avons dit : « nous sommes en train d'aller, nous sommes en train d'aller, nous sommes en train d'aller chez eux, ils sont là ", j'ai dit.

Dans cet endroit où les Blancs habitaient, il y avait deux maisons; un groupe allait dans une direction, nous attendions dans l'autre ; je regardais face à moi pour voir s'il y avait quelque personne blanche afin de la poursuivre et la tuer.

Le premier groupe qui partit, partit vers l'autre maison. Ils rentrèrent dans la maison et Ibicaidé fut l'Ayoré qui tua la première femme blanche, qui la tua à cette occasion-là. Après c'est Hoedé ou Padodi qui tua l'autre dans le même endroit. Je suis allé autour de l'autre maison parce que j'avais peur que quelqu'un puisse s'échapper, je suis allé directement, mais quand je me suis aperçu que là il n'y avait personne, je commençai à courir vers l'autre maison où étaient mes camarades.

Je suis allé vers eux au moment où ils étaient déjà en train de tuer quelques femmes blanches, je suis allé et j'ai dit comme ça : « je vais déjà, je vais déjà, je vais déjà chez eux, je suis en train d'aller vers eux ", je disais comme ça à mes camarades.

À ce moment Hoedé tira une lance contre cette femme, mais il n'arriva pas à la tuer ; c'est Eidaqui, ou bien Oiadaquidé, qui regarda la victime par terre et la coupa jusqu'à ce qu'elle meure. Hoedé revint et dit : « c'est moi qui l'ai tuée, c'est moi qui l'ai tuée » et j'ai dit « oui, oui, oui c'est toi qui l'as tuée, mais moi je vais tuer son fils ».

À ce moment, quand la femme était en train d'expirer, je criai aux jeunes : « jeunes, jeunes, il faut tout prendre, tout ce qui appartient à cette famille, cherchez, cherchez », je dis et je continuai.

Quand je suis rentré dans les pièces de l'endroit où ces gens vivaient, j'ai vu deux lits et encore d'autres choses qu'ils avaient. À ce moment quand je suis rentré, j'ai baissé ma lance et j'ai pris les choses, couvertures, linges et donc je suis parti de cet endroit, je suis parti rapidement et j'ai couru. À ce moment quand je suis parti de la maison, j'ai couru vers la forêt et j'ai emmené avec moi les choses, c'est-à-dire des couvertures, des linges et bien d'autres choses que j'avais enlevées.

Cependant aucun de nos camarades ne revenait, ils étaient en train de se charger de choses et également de haricots et d'autres aliments des Blancs, mais les haricots, ils les ont reconnus parce qu'ils savaient déjà même le nom. Quand nous arrivâmes chez d'autres camarades, nous avons dit : « cependant, les autres ne sont pas encore arrivés ». 
À ce moment, chacun de nous mit ensemble la tê te des victimes tuées à cette occasion-là. Et, tous ensemble, nous nous sommes retrouvés dans l'endroit où l'on avait établi de se rencontrer. Dans cet affrontement, nous avons tué deux femmes et deux filles, alors que deux garçons qui étaient en train de jouer dans la forêt se sont sauvés. Ici s'achève le récit de cette tuerie de Blancs.

Le troisième récit est raconté par Paojnai, du clan ciquenoi, qui a décidé, il y a quatre ans, avec sa femme, Iboé ${ }^{12}$, et ses six enfants de prendre contact avec les Blancs :

À ce moment-là, nous étions en train de poursuivre un tapir (cabayú $\left.{ }^{13}\right)$ de la forêt. Nous étions en train de nous approcher du tapir et mon épouse cria à ce moment d'un autre endroit et je lui dis : " nous le voyons, nous le voyons, le tapir ».

À ce moment, mon épouse le siffla et le tapir s'arrêta et moi avec ma lance, j'allai à la rencontre du tapir.

Quand le tapir fut dans notre direction, je dis : « le tapir va nous attraper, le tapir va nous attraper $"$, je dis comme ça à mon épouse et à mes camarades.

Et quand le tapir m'attaqua, je le transperçai sur le côté et je criai : « je l'ai transpercé, je l'ai transpercé » et, avec mon bâton de défense, je courus derrière lui pour le frapper bien fort sur la tête.

À ce moment, mes camarades avaient couru derrière le tapir et l'avaient frappé ; ils l'avaient tué un peu plus loin et moi, à ce moment, j'avais déterré avec mon bâton une espèce de tubercule qui contenait de l'eau car je voulais en tirer de l'eau pour boire. Quand j'étais en train de déterrer ce tubercule dans lequel il y avait de l'eau, j'entendis une rumeur qui venait vers moi ; à ce moment je me suis levé et j'ai regardé devant moi et j'ai vu qu'il s'agissait d'un jaguar.

Je lui ai dit : « venez vers moi, venez vers moi, venez vers moi », je n'ai pas peur ; et donc il s'arrêta loin de moi, et moi je voulais lui envoyer ma lance comme ça au jaguar.

Alors, à ce moment, je dis à mon épouse : « j'y vais, je vais chercher le jaguar », et je suis allé le chercher. J'étais donc loin du campement et je vis les traces du jaguar et je me dis à moi-même : " c'est le jaguar, c'est le jaguar, j'irai derrière lui ", je dis comme ça.

Et quand je le vis, je m'approchai et je lançai ma lance, je lançai ma lance dans le cou du jaguar et quand je le transperçai, il fit ce bruit : « hee, hee, hee ", il fit comme ça.

Et à ce moment-là après avoir transpercé le cou du jaguar, j'ai utilisé ma dague et je coupai la tête du jaguar et je me dis à moi-même : «j'ai tué un jaguar, un jaguar très grand », sa tête aussi était très grande.

Voici le récit du jaguar que j’ai tué.

En dépit de leurs contenus distincts (sur la guerre proprement dite, la tuerie, la chasse ou la rencontre fortuite avec les jaguars), ces récits partagent les mêmes registres gestuels et vocaux. Les récits ayorés de guerre ainsi que ceux de mises à mort ont un caractère essentiel qui est commun à toutes les expressions de leur culture, notamment orale. Ainsi, la présence d'éléments contextuels est rare, la redondance est strictement orientée vers des formules destinées à mettre en valeur l'habileté demandée aux guerriers. Nous observons dans ces récits l'alternance de 
deux moments qui trouvent des correspondances significatives dans d'autres registres de la vie sociale. D’une part, le récit procède selon un style récitatif, marqué par des tons et des gestes dont la variété n'est due qu'à l'exécution particulière de chaque conteur, tout en puisant dans les mêmes codes expressifs. Parmi ces gestes, signalons : la lance plantée dans la terre pour marquer le début et la fin de chaque histoire, le geste du jaguar en train de sauter ou bien la lance tournée vers le haut pour indiquer que celui-ci s'est réfugié sur un arbre, la lance qui est soulevée pour frapper ou bien enfoncée dans l'ennemi déjà mort, la pointe de l'arme retournée vers soi-même pour indiquer une blessure. D'autre part, la narration connaît des accélérations soudaines, qui sont réalisées avec la voix et le corps encore plus tendus et qui soulignent par des mots répétés et par des gestes canoniques en style représentatif (pourrait-on dire en langage musical) les mouvements rapides dans l'espace : surtout ceux de départ, d'attaque ou de retour lorsque l'action de guerre est terminée.

Cette accélération - qu'en utilisant un concept de Jauss repris par Ong (1982) nous pourrions appeler verbomotrice - consiste en une torsion légère de la main droite qui tient la lance que l'on fait glisser sous l'aisselle dans un mouvement d'avant et d'arrière, pendant que l'autre bras, main ouverte ou fermée, est tendu dans la direction contraire. En même temps, le pied, d'abord soulevé plus ou moins lentement, retombe pesamment par terre dès que le bras qui ne tient pas la lance est complètement tendu. Lorsqu'on utilise deux armes, celle qui est dans la main gauche est tenue parallèle au sol. Un détail significatif : tous les conteurs, s'ils utilisent une seule arme pour le récit, portent leur main sur la cuisse, puis la tirent vers le haut; la cuisse dont d'aucuns Ayorés soulignent l'importance comme siège de force et de résistance. Le geste (Figures 2 à 5), propre à une attitude épique dont l'histoire est encore à faire ${ }^{14}$, représente une espèce de " marche furieuse » menée « de corps ferme », pourrait-on dire, où le mouvement des jambes et des pieds qui marquent le pas donne unité et rythme à l'ensemble. Notons que le conteur utilise aussi assez souvent d'autres tons pour s'adresser directement à son public, pour lui faire peur, ou bien pour mimer une communication à distance dans la forêt.

Dans le cadre du récit, le geste qui exprime le mouvement est autant valorisé que celui de tuer, ce qui est compréhensible dans une société où la période de nomadisme polycentrique (saison appelée "le monde fermé ») est idéologiquement aussi importante, sinon plus, que celle de sédentarité (appelée siquée, «l'année », terme qui indique aussi l'ensemble des produits cultivés), tout au moins par rapport aux épreuves que la nature impose aux chasseurs-cueilleurs. Cependant, si cet état d'hostilité qui caractérise la culture ayoré s'intensifie particulièrement pendant la période de nomadisme, atteignant un acmé lors de la convergence de différents groupes vers les salines qui se trouvent au centre de leur territoire à la frontière entre le Paraguay et la Bolivie, le bellicisme n'en est pas moins significatif pendant la période des campements permanents. En tout cas, 


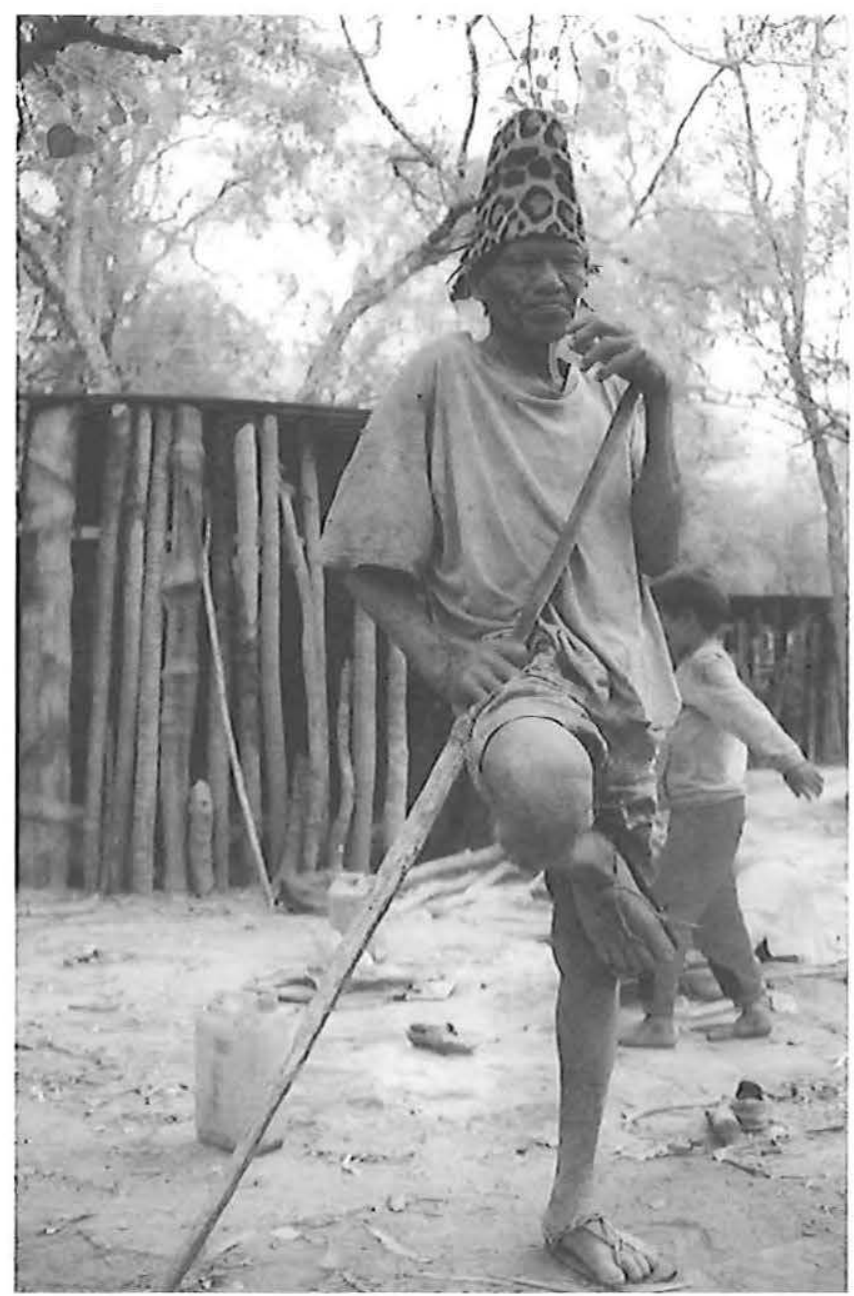

FIG. 2. - Aocójnandi, guerrier au repos (cliché D’Onofrio 2001)

aucune des deux périodes ne l'emporte sur l'autre, même du point de vue économique, car, justement, ce n'est que l'articulation des deux moments qui fait sens.

L'alternance que nous venons de voir n'est pas un fait isolé dans la culture ayoré. Celle-ci nous offre un modèle exemplaire d'« orientation dualiste » qui relie entre eux plusieurs registres de la vie sociale par des « enchaînements symboliques élémentaires » dont l'homme n'est que l'intermédiaire inconscient. 

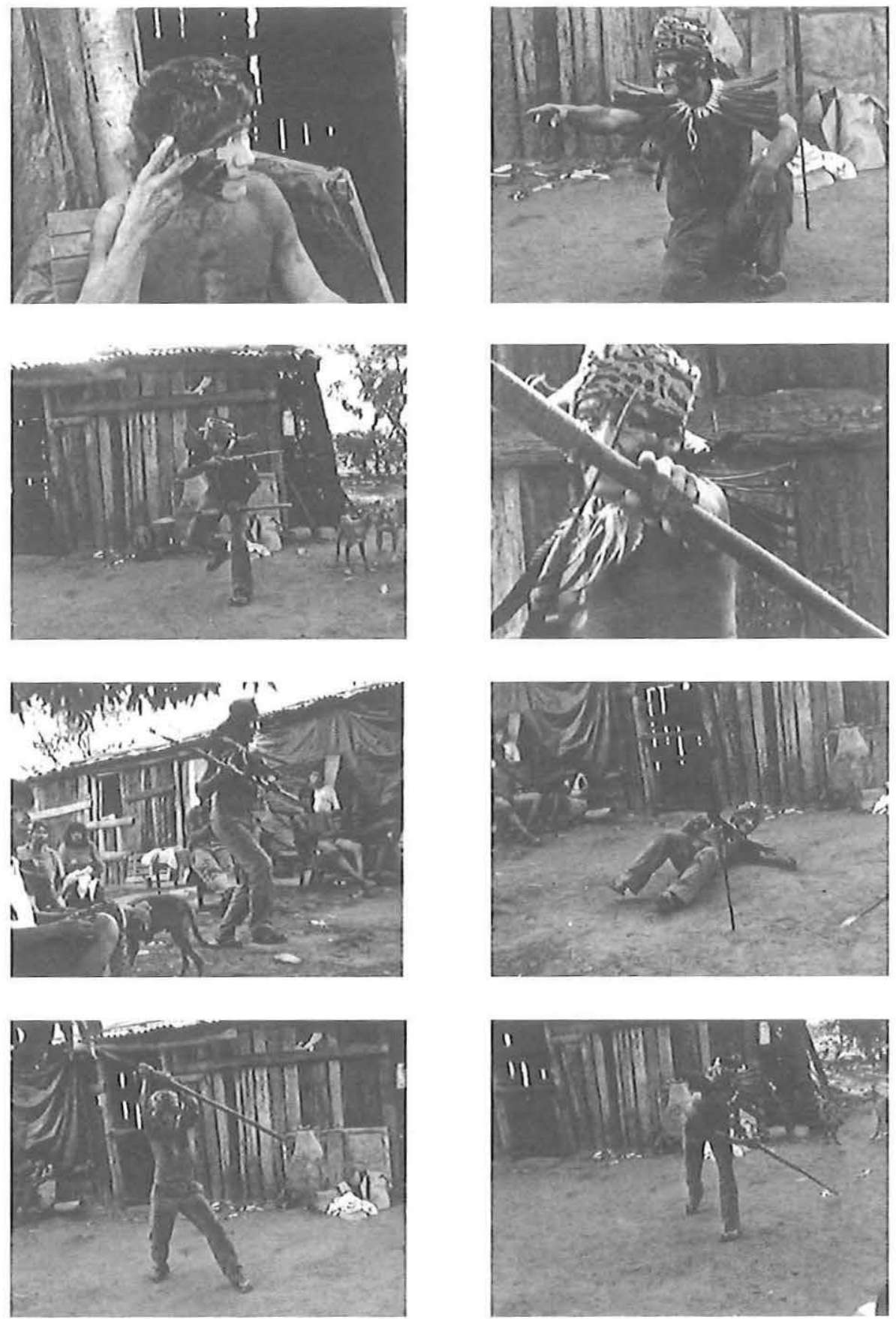

FIG. 3. - Cucaani, séquence d'un récit de guerre (clichés D'Onofrio 2000. Lecture de gauche à droite) 

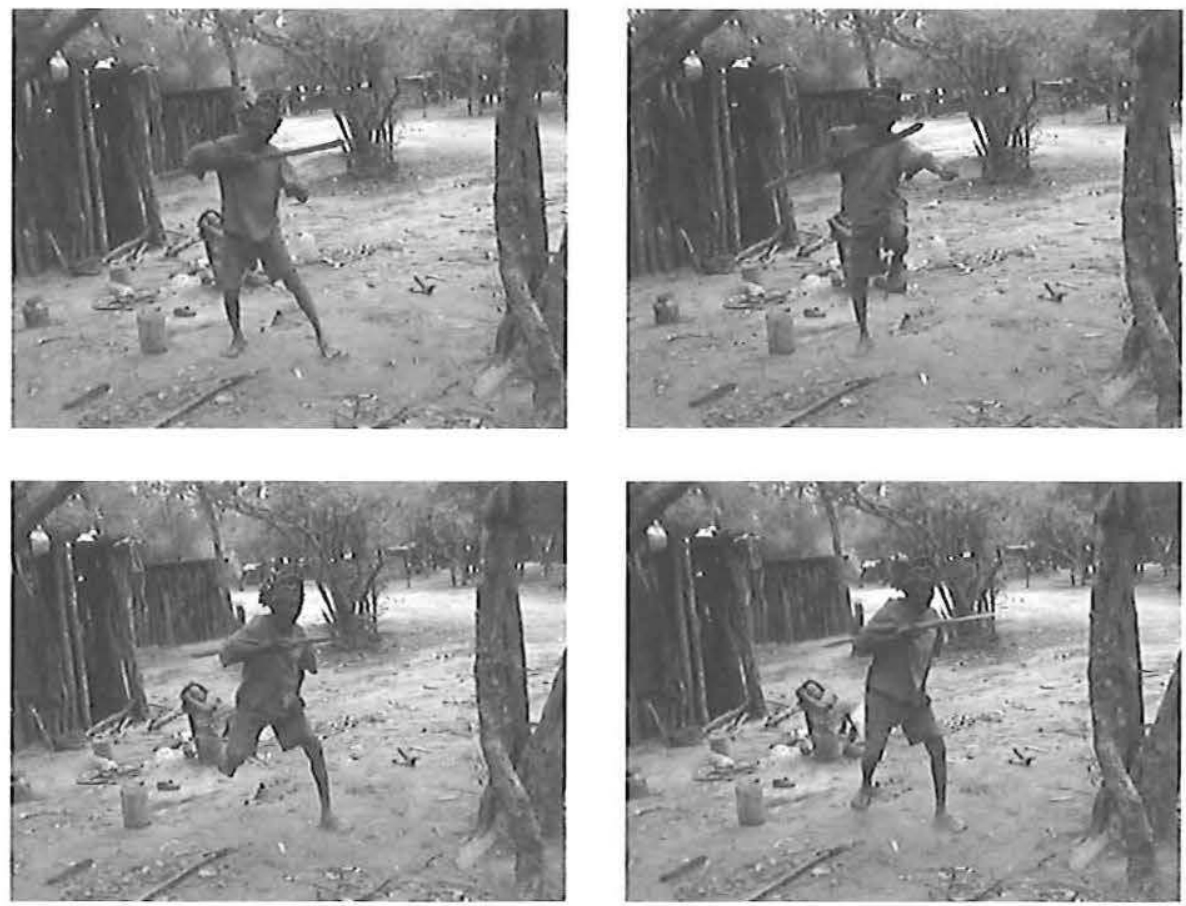

FIG. 4. - Aocójnandí, récit d'une lutte contre un jaguar (clichés D’Onofrio 2001. Lecture de gauche à droite)

Ces " enchaînements ", comme le montrent souvent les mythes, se déroulent à partir d'oppositions binaires telles que : masculin-féminin, jour-nuit, cru-cuit et leur automatisme est assuré par le même mécanisme qui règle la formation des signes. Ce qui n'empêche évidemment pas que l'homme soit toujours au centre car, en dernier ressort, il est l'origine et le garant de la circulation et de la compatibilité des signes au sein d'un système.

L'analyse de la peinture faciale permet de saisir d'autres aspects de cette orientation dualiste de la société ayoré, tout en montrant les correspondances entre la guerre et les activités de reproduction qui traversent les générations et les sexes. Seules les jeunes filles (et les petites filles) peuvent tracer sur leur visage les dessins traditionnels en rouge et noir. Cette pratique est complètement abandonnée dès lors qu'une relation sexuelle temporaire est transformée en une relation matrimoniale stable. Mais elle est réutilisée lorsqu'il s'agit d'une jeune femme séparée.

La peinture faciale oppose donc deux époques de la vie complètement distinctes : d'une part, l'adolescence qui peut se prolonger jusqu'à dix-huit ou vingt ans et qui est marquée par la liberté sexuelle et la joie de vivre ; d'autre part, l'âge adulte marqué par des comportements rigoureux et austères qui ont poussé 

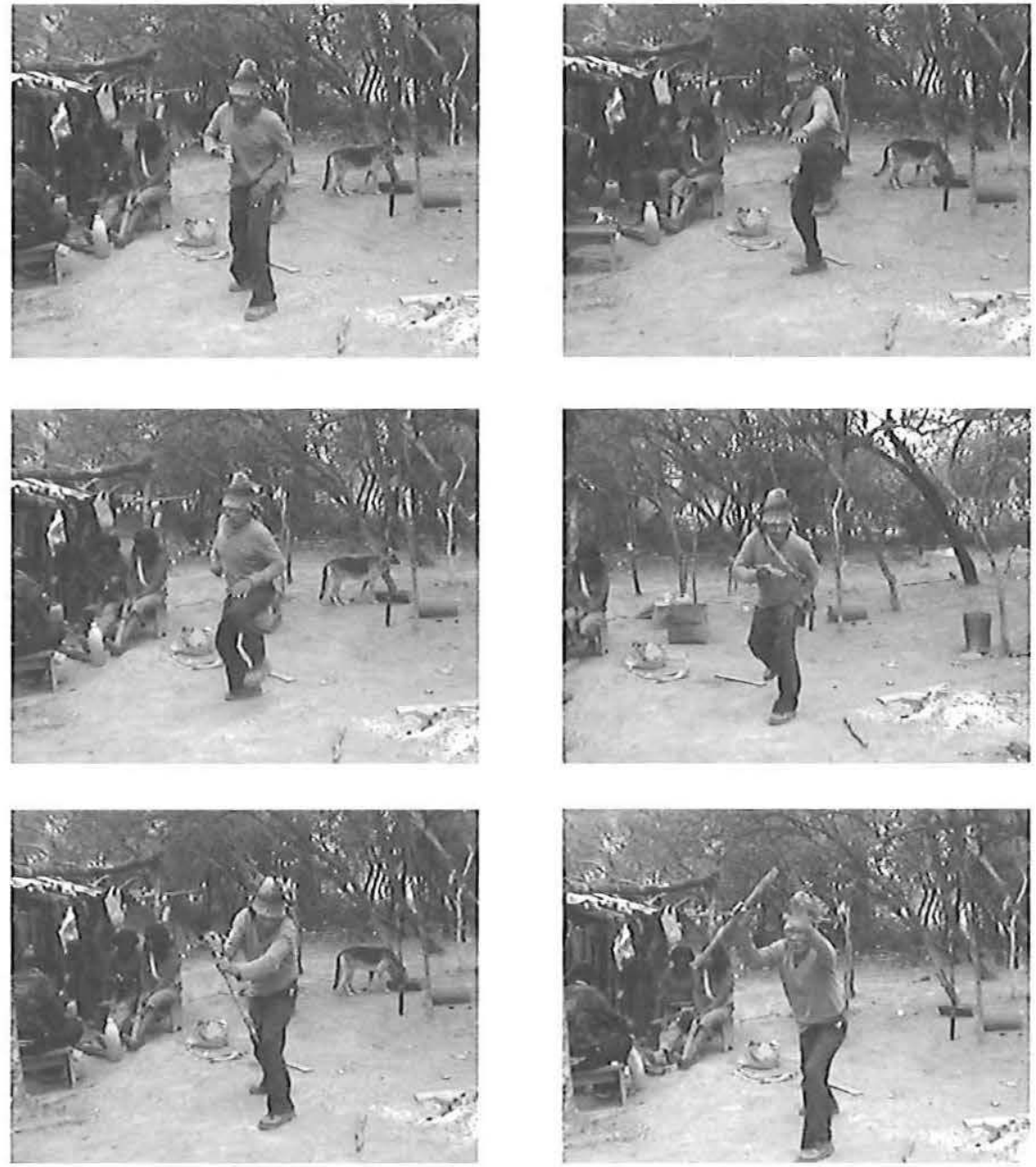

Fig. 5. - Campo Loro, séquence d'un récit de guerre (clichés D’Onofrio 2001. Lecture de gauche à droite).

quelques chercheurs à parler de « culture apollinienne » débordant même dans le «tantrique ». En réalité, l'opposition apollinien/dionysiaque de la Grèce ancienne ne se justifie qu'en rapprochant ces deux termes des attitudes qui opposent et relient ces deux âges de la vie. Des incursions d'attitudes en principe propres à la jeunesse sont pourtant admises dans le monde des adultes, qui connaît par exemple des moments de gaieté pétillante ainsi que le rôle, très apprécié par les femmes, du personnage pitre. 
Le registre sexuel est aussi important que le registre générationnel. Chez les Ayorés, le choix du conjoint, comme celui des partenaires sexuels prématrimoniaux, revient exclusivement aux femmes. En forêt, les jeunes filles allaient chercher les garçons là où ils avaient l'habitude de vivre, au centre du campement qui était appelé aussi « le lieu de la musique ». Et parfois, encore maintenant, il peut arriver qu'une jeune fille soit aidée par ses amies pour convaincre et même " enlever» un garçon afin de l'emmener avec elle chez ses parents (puisque, comme nous l'avons vu, les Ayorés sont uxorilocaux). En d'autres termes, des évidences et des enchaînements symboliques élémentaires montrent que la quête d'amour et la guerre sont perçues comme étant très proches. "Chercher l'homme » est assimilé du point de vue idéologique à l'activité prédatrice, une prédation culturellement tout aussi contrôlée que celle assumée par l'homme vis-à-vis du gibier et de l'ennemi ${ }^{15}$.

Les jeunes filles ne recourent pas, comme les guerriers, à la peinture intégrale du corps, alors que les garçons, s'ils le veulent, peuvent eux aussi peindre leur visage dans l'attente des jeunes filles qui leur imposent, plus qu'elles ne sollicitent, leur intérêt sexuel. Mais, seuls les hommes peuvent continuer à se peindre en tant que guerriers, et cela jusqu'à ce que, devenus grands-pères, ils ne soient plus à même de chasser et de se battre. Le changement sera alors souligné par l'usage d'une triple ficelle de cáaguatá qui entoure les flancs et qui se substitue au cache-sexe en plumes ou en tissu de cáaguatá et par l'usage plus fréquent du pámoi, une bande de cáaguatá qui entoure les flancs et sert à maintenir surtout les genoux quand on est assis. Significativement, un homme qui a été blessé en guerre peut utiliser le pámoi car il est classé dès lors parmi les vieux.

Pendant les amours juvéniles, une sorte de « contamination chromatique » est pourtant possible, car les hommes peuvent parfois utiliser eux aussi la couleur rouge qu'habituellement les femmes utilisent pour tracer des lignes partant en général des extrémités latérales des yeux et des angles de la bouche (Figure 6) ${ }^{16}$. Il en va de même pendant la fête d'Asoojná, au retour de l'expédition de chasse et de récolte de miel en forêt et après avoir pris le bain et rompu le jeûne qui durait depuis la nuit précédente. Mais, dans ce cas-là, cette contamination marque justement le passage de la forêt au campement et l'effacement de l'interdit des relations sexuelles qui reprendront très librement au lever du soleil.

La couleur des guerriers est le noir et le blanc sale des dessins obtenus en passant le doigt mouillé de salive sur le noir de fumée. À partir de la diversité sexuelle, nous retrouvons ainsi au niveau des couleurs une articulation significative, confirmée par les mythes : la couleur féminine rouge qui renvoie au plaisir et à la vie, la couleur masculine noire qui renvoie à la tuerie et à la mort. Le rouge et le noir (qui est parfois un bleu) sont, avec le blanc, les couleurs des sacs de cáaguatá, dont les motifs se fondent sur les marques des sept clans ayorés.

Ajoutons enfin que les ornements utilisés durant la guerre et lors du récit sont strictement interdits aux femmes et il revient à l'homme de les transporter dans 

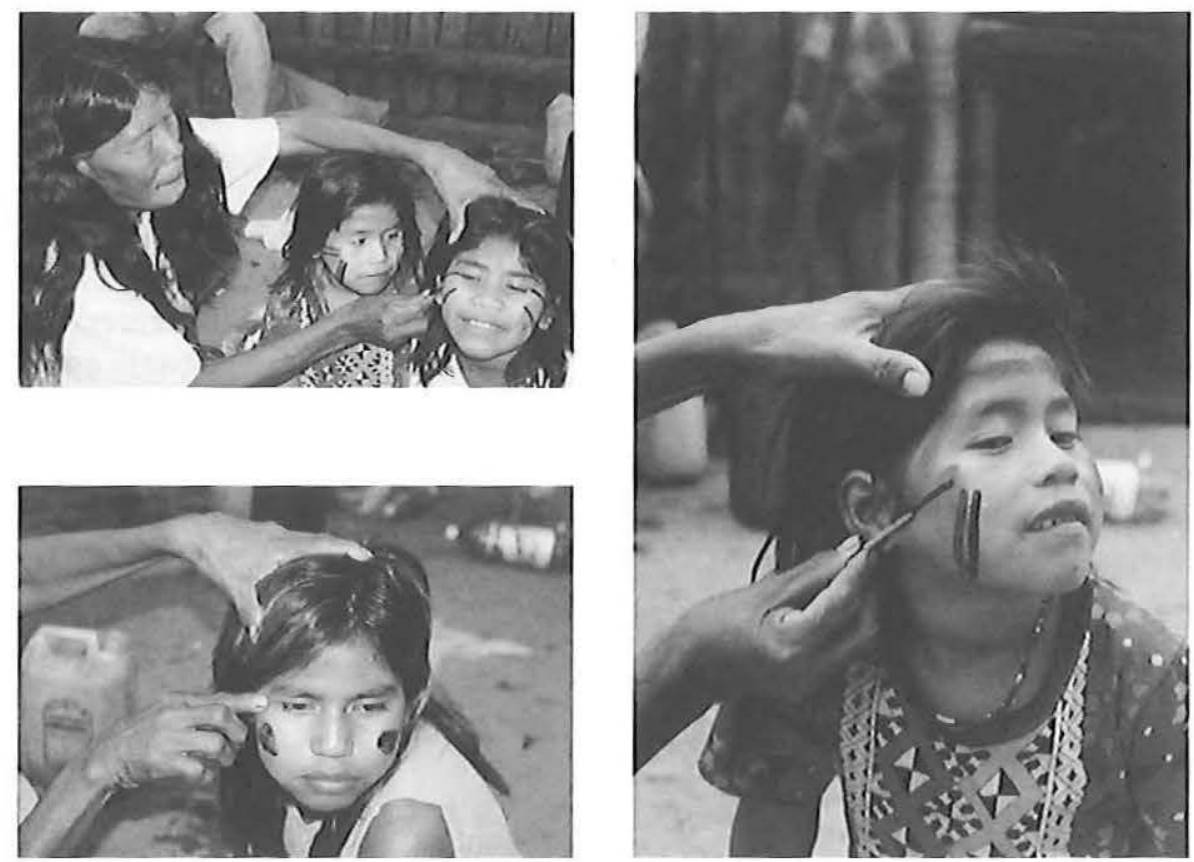

Fig. 6. - Cucaani, peintures faciales (clichés D’Onofrio 2000)

des sacs spéciaux (poapi) que les femmes tressent mais qu'elles n'ont plus le droit de toucher dès qu'ils ont été rendus puyác ("sacré ») par le contact avec ces ornements.

Soulignons encore la valeur mythique de la parole dans le cadre de ce genre de récits. Combinée au geste, la parole présente d'abord, dans les accélérations qui expriment le mouvement, cette « triple répétition » qu'au début du $\mathrm{xx}^{\mathrm{e}}$ siècle Axel Olrik (1973) avait découverte dans les séquences d'événements qui structurent les fables, répétition dont Lévi-Strauss a bien montré l'importance dans les mythes. Mais il y a aussi des différences dont il faut tenir compte entre ces deux genres. Dans les récits de guerre, on ne trouve pas le double niveau de signification qui caractérise les mythes (la valeur différente des mythèmes dans le récit et dans le contexte ethnographique). Par ailleurs, leur lecture paradigmatique, pourtant parfois possible, ne présente pas de significations particulières au regard de ce que ces récits nous disent du point de vue syntagmatique, au fil de la narration. C'est ailleurs qu'il faut en chercher la structure. Notamment dans la redondance, dans la canonicité des gestes et, surtout, dans l'usage spécifique de la parole. Dans les récits de guerre, la parole n'est pas accompagnée - contrairement à celle du chamane lorsqu'il l'utilise en situation thérapeutique - par d'autres éléments venant du dedans, la salive ou le souffle par exemple. En revanche, elle est 
prononcée pour confirmer le courage ainsi que le statut de tueur du guerrier, statut qui confère occasionnellement un pouvoir normalement réservé au chamane (naijnai), à savoir la possibilité de parler directement avec les plantes, les animaux et les morts.

Comme le chamane, le guerrier ayoré possède donc des pouvoirs qui ne sont accordés qu'à une certaine catégorie de personnes et qui actualisent l'état mythique originaire, ou bien le passage de celui-ci à l'état présent. Ce pouvoir créateur de la parole se manifeste dans les moments qui précèdent la guerre, et dont nous donnerons quelques exemples, ainsi que dans ceux qui la suivent. Une considération intégrale de ces moments montrera par ailleurs un aspect paradoxal que nous nous limitons à indiquer : le paradoxe d'une société qui, d'une part, semble ne pas avoir besoin de justifier la guerre pour la mettre en acte (car quelques récits disent clairement qu'il ne s'agissait pas de vengeance ou, du moins, pas autrement que la simple envie de tuer) et qui, d'autre part, élabore toute une série d'apparats formulaires, rythmiques et gestuels qui soustraient l'événement à l'histoire et le rendent pratiquement possible tout en l'inscrivant en même temps dans l'horizon du mythe. En d'autres termes, s'il est vrai que les Ayorés peuvent tuer sans aucune raison, tout au moins apparente (car, en réalité, ils distinguent bien par exemple à l'intérieur de leur propre ethnie les ennemis des alliés), il est également vrai qu'il est impossible pour eux de tuer tout simplement. Ils sont obligés de le faire symboliquement avant et après la vraie guerre.

Un aspect significatif de cette efficacité symbolique liée à la parole est la croyance dans le fait que la guerre peut se déclencher à la suite d'un simple récit de mythe, par exemple celui de l'origine du tatou (ajáamei) ou celui du foyer ${ }^{17}$. D'où la prohibition de les raconter qui est d'autant plus frappante que sa transgression est censée provoquer la folie du conteur et qu'elle s'applique à un objet culturel, le mythe, dont l'existence n'est garantie que par sa transmission à travers le récit. À ce propos, deux points méritent d'être soulignés. En premier lieu, la prohibition (puyác) - étant un concept qui exprime une idée de puissance autant que d'impureté - doit son efficacité justement au fait qu'elle est négociable, comme le découvrent aussitôt tous ceux qui font des enquêtes sur la mythologie ayoré : certains mythes ne peuvent se raconter qu'en dehors du village, d'autres à des moments particuliers, ou bien en en omettant quelques parties, etc. En deuxième lieu, par cette prohibition et par d'autres éléments du discours sur la guerre (par exemple des signes qui l'annoncent, tel le chant d'une chauve-souris, chaboto), il apparaît clairement que celle-ci n'est pas, en général, vraiment souhaitée. Bien qu'elle ne soit pas un événement isolé ni exceptionnel, la guerre ne fait pas l'objet d'une recherche acharnée, mais plutôt elle est une ressource permanente et disponible, le cas échéant, à des fins diverses. Pour pouvoir faire appel à cette ressource, il faut la justifier dans l'horizon mythique. C'est le seul trait vraiment unifiant de la guerre chez les Ayorés. Celle-ci n'est d'ailleurs l'effet d'aucune autre détermination générale ; elle s'inscrit cependant à part entière à 
l'intérieur du champ social qu'elle-même contribue à structurer ou à réactiver. Comme l'a bien écrit Philippe Descola (1993) pour l'ensemble de la guerre amérindienne, nous voyons plus aisément dans la guerre chez les Ayorés « une manifestation spécifique de certains types de rapports sociaux à travers lesquels l'identité, les frontières ethniques et les positions statutaires sont constamment négociées et reproduites » (ibid., p. 172).

La guerre imprègne donc de son idéologie non seulement la presque totalité des rapports sociaux mais aussi toute une série d'actes rituels et symboliques qui doivent avoir lieu pour qu'elle puisse être efficace aussi au niveau de la praxis. Passons donc aux exemples qui confirment, par leur place dans la séquence où ils apparaissent, la logique que nous venons d'exposer: la vision chamanique (enominone), l'emportement (chugui), les chants qui précèdent et ceux qui suivent le combat, la purification (páagápidi).

\section{LA VISION}

En ce qui concerne la vision chamanique, nous nous limiterons à rapporter quelques textes et à signaler que le chamane doit parfois rendre compte du mauvais résultat d'une guerre dont on le tient pour responsable, sauf s'il est en même temps un chef. Tel est le cas d'un des chefs les plus importants des Ayorés du groupe guidáigosode, Uejái ${ }^{18}$, du clan picaneai, auquel on doit la première vision, chantée à la troisième personne par Cájode, un de ses lieutenants qui habite actuellement dans la communauté de Campo Loro, à $80 \mathrm{~km}$ de Filadelfia dans le Chaco central :

Uejái dit à une de ses épouses dont le nom est Atequeuadaté : « Lève-toi et écoute ce qui est dit dans ma vision : les Blancs et nous, nous allons nous rencontrer; les jeunes obtiendront les armes à feu des Blancs pour disperser les autres Ayorés. La deuxième génération de jeunes vivra avec les Blancs, mais nous, les premiers pères, nous allons mourir à cause des maladies. Après ça, nous allons avoir aussi des relations avec les Totobiégosode et, après, dès que nous vivrons avec les Blancs, nous allons avoir encore des affrontements entre nous $»$.

Dans cette vision, le grand chef Uejái fait référence à l'inévitable rencontre avec les Blancs, ce qui l'amène à voir non seulement les maladies mais aussi la possibilité de se battre contre leurs ennemis traditionnels, les Ayorés du groupe totobiégosode (litt. : « ceux qui vivent là où abondent les pécaris »), en ayant les armes des Blancs, pour après se réconcilier et recommencer à se battre contre eux. Dans les faits, des expéditions et des combats entre ces groupes ayorés se sont poursuivis, souvent avec la complicité des missionnaires, jusqu'aux années 1980 (précisément, jusqu'en 1986). Cette complicité s'est même parée d'un fondement théologique. Les missionnaires nord-américains de $A$ Las Nuevas Tribus avancent 
la théorie, par exemple, que la foi des Ayorés qui vivent dans les missions sera toujours mise en danger tant qu'il y aura des Ayorés vivant en forêt et qui n'ont jamais eu de contacts avec les Blancs.

La vision chantée par un autre chamane du groupe guidáigosode de Campo Loro, Mariadé-Oji ${ }^{19}$, concerne elle aussi la guerre et les maladies qu'elle provoque, ainsi qu'un geste rituel de purification effectué par la mère du tueur :

Le corbeau m'appela et me dit : «neveu, sais-tu ce qu'est ce que je tiens dans ma main? » Je lui répondis en disant qu'il s'agissait d'un cheveu d'Ayoré qu'il tenait dans sa main. Apparemment, mon oncle avait tué quelqu'un parmi les Ayorés. Judui lui aussi me parla en me demandant : "neveu, sais-tu ce qu'est ce que je tiens dans ma main ?» Je lui ai répondu en lui disant que ce qu'il tenait dans la main était du sable. Il répondit que j'avais deviné et il continua : « moi, j'ai déjà enterré deux de leurs chefs qui sont morts. Mon frère, si nous retournons au campement, Usuguede et Jongoiné seront à l'entrée du campement à nous attendre et ma mère prendra une poignée de terre et la lancera derrière nous. Ma mère va lancer cette poignée de poudre de terre quand ils crieront à haute voix. Comme ça, nous ne mourrons pas d'une maladie ».

La dernière vision, toujours chantée par Mariadé-Ojí, est celle d’Uodáquidé, un Ayoré du groupe gaáigosode, mort avant le contact avec les Blancs ; elle aussi concerne la guerre entre Ayorés ainsi que la paix avec les Blancs :

Ioguedatea, nous irons trouver quelque part les os de notre oncle, mais il ne faut pas toucher ces os.

Si quelqu'un touche ces os, une maladie se transmettra à celui qui touche les os, comme le dit l'oiseau (chunguppejná).

Je lui dis de se lever et de réveiller aussi un de ses fils pour qu'il le suive à Ichaguedáquidé car, demain, les Blancs vont tuer Iguadigué et ils tireront beaucoup de projectiles.

Je fis attention à ceux qui étaient en train de crier, mais ceux qui criaient étaient les choses que nous avions oubliées sur les territoires qui nous appartenaient.

J'ai vu aussi ton frère Acolite, mais j'ai vu que les Ayorés vont se tuer entre eux, mais tes frères vont leur échapper, et ton oncle et ton frère Acolite vivront avec les Blancs.

Seulement eux vivront avec les Blancs.

Si quelqu'un parmi vous a de bonnes oreilles, qu'il écoute bien et qu'il reste avec nos oncles et nos frères.

\section{L'EMPORTEMENT}

Nous retrouvons la même conjonction avec les choses, telle qu'elle est exhibée dans la troisième vision, dans le chugui, le rituel que les guerriers exécutent à la suite de l'annonce de la guerre par le chef. Cette annonce se fait après la vision éventuelle, après que le chef reconnu comme le plus important a organisé une réunion avec les chefs des campements voisins, du même groupe ayoré ou de groupes alliés, pour planifier la guerre ${ }^{20}$. 
Le rituel est le plus souvent individuel, bien que l'on puisse parfois le faire collectivement, au cours d'une cérémonie plus complexe dont nous parlerons plus loin. Les guerriers secouent leurs corps - ce qui leur permet parfois d'atteindre un véritable état de " transe nerveuse »- et poussent des cris qui ressemblent plutôt à des invocations dramatiques. Par ces cris, ils peuvent montrer qu'ils n'ont pas peur et afficher leur mépris de la mort, tout en se représentant en quelque sorte comme déjà morts et en s'assimilant à des éléments de la nature : des plantes, des animaux ou des corps célestes. Chacun choisit un élément parmi ceux qui appartiennent à son clan, les edópasade ${ }^{21}$. Il s'agit de véritables "gisements symboliques " grâce auxquels les clans se réfèrent les uns aux autres et qui constituent la base des " enchaînements symboliques élémentaires » que nous avons postulés. Le terme utilisé pour cet emportement, chugui, est significatif en soi car il indique dans son sens premier le bourdonnement furieux des abeilles quand les Ayorés récoltent le miel des ruches.

Nous avons analysé le contenu du chugui dans les groupes gaáigosode, guidáigosode et totobiégosode du Chaco central. Bien qu'ennemis entre eux les guerriers de ces groupes proposent des identifications aux mêmes éléments puisque les clans auxquels ils appartiennent sont les mêmes.

Commençons par le clan chiquenoi qui est le plus important non seulement du point de vue démographique mais aussi mythique, car il est le seul dont on dit qu'il transgressa à l'origine la prohibition de se marier à l'intérieur de son clan. Le guerrier chiquenoi s'identifie avec le jochacai (une espèce de tatou) qui est un des edópasade du clan chiquenoi. Le choix est fait en raison de la dureté de la carapace de cet animal, de sorte que les coups de l'ennemi n'ont pas d'efficacité sur le guerrier chiquenoi. Celui-ci a donc la peau dure.

Le guerrier etácoé s'assimile au soleil, guedé, en fonction de la chaleur qui émane de celui-ci et qui empêche l'ennemi de s'approcher. Pour la même raison, il peut aussi choisir l'assimilation au feu, piói. De même que chez les Chiquenoi, cette identification montre que la préoccupation principale des Ayorés est d'empêcher que l'adversaire ne s'approche et n'attaque par surprise (parfois avec des cris provoquant la terreur) : il s'agit de la technique de guerre à laquelle on a le plus fréquemment recours.

Les guerriers picaneane choisissent un oiseau mythique, le quiaquia, un faucon charognard que les Ayorés, bien qu'ils en utilisent les plumes pour leurs colliers, considèrent comme un animal très sale et impur dont ils n'aiment pas s'approcher. D'après la description des attitudes de cet oiseau, il s'agit sûrement d'un charognard. Les deux dictionnaires ayorés que nous avons consultés donnent : " carancho, quiraquira » (Barrios, Bulfe et Zanardini 1995, s.v. carancho [fr. caracara]) ; « hawk/buzzard [fr. faucon/busard] that feeds on carrion » (New Tribes Mission 2000, s.v. quiráquirái). Ce dictionnaire précise dans une note : « when full-grown, the quiráquirái has black body feathers, white edges on wings, black on top of head, and red around beak ». Noir, blanc et rouge sont bien les trois 
couleurs de base des Ayorés que l'on retrouve dans les sacs de cáaguatá et non par hasard sur le corps de cet oiseau qui est parmi les plus importants de la mythologie ayoré. C'est quiaquia qui a permis aux Picaneane d'imiter sa peinture pour peindre les motifs de leurs sacs de cáaguatá. En principe ils sont les seuls à utiliser deux par deux les quatre combinaisons qu'il est possible d'obtenir avec ces trois couleurs, ce qui montre comment la culture ayoré définit son unité ethnique au moyen du style qui est propre à chacun de ses sept clans et qui remonte au temps des origines. En s'identifiant à cet animal, le guerrier ayoré veut donc empêcher son ennemi de s'approcher. Mais il y a plus. Implicitement, il traite celui-ci de charogne, c'est-à-dire de personne n'ayant pas eu droit à l'enterrement car il est classé parmi ceux à qui on a coupé la tête à la bataille. On reviendra plus tard sur le traitement de la tête de l'ennemi.

Le guerrier dosapei choisit le petangai, une variété de papillon qui se lance dans le feu jusqu'à en mourir. C'est par cette identification que les dosapéode expriment leur mépris de la mort.

Le choix des Cutamuajane est orienté vers le quebracho rouge (tujnini) car, comme disent les Ayorés, "il est déjà sur le feu ». Essentiellement, il y a trois raisons pour lesquelles cette plante est choisie : sa dureté, sa couleur - qui permet de l'assimiler à un des éléments empêchant l'ennemi de s'approcher, c'est-à-dire le feu - et ses étincelles produites au contact du feu lorsqu'on lui souffle dessus.

Le choix des Posóajane est double et montre la volonté des Ayorés d'intégrer dans leur horizon mythique des éléments propres à la culture et au monde naturel de leurs ennemis. Le guerrier posóajá choisit traditionnellement de s'identifier au cochon sauvage (ñacoé), car celui-ci, furieux, empêche son adversaire de rentrer dans son groupe. Comme on l'a vu pour la chaleur, la dureté de la peau et la saleté, la furie de cet animal empêche l'ennemi de s'approcher. Le choix est si important qu'on souligne non seulement la difficulté de rentrer dans le groupe de cet animal mais aussi sa furie, qui est analogue à celle que le guerrier atteint par le rituel dit chugui. De plus, le cochon sauvage est déjà furieux, exactement comme le guerrier ayoré qui garde cet état de rage pendant toute la période précédant la recherche et l'attaque de l'ennemi. Dans ce cadre, une signification spéciale est accordée à l'effervescence sexuelle qui caractérise les déplacements vers l'ennemi et qui se manifeste dans la licence plus ample accordée aux accouplements entre les jeunes filles qui suivent le groupe et les guerriers déjà mariés. La deuxième possibilité du guerrier posóajá est tournée vers un animal des Blancs, la vache, aquíajna en ayoré. Les Ayorés, qui rapprochent cet animal du tapir, l'ont inscrit dans leur propre horizon mythique. Il en est de même pour d'autres éléments du monde occidental qu'ils découvraient pendant les phases de repérage, de contact et de colonisation. Le rapprochement avec le tapir est extrêmement significatif, car la vache apparaît aux Ayorés vivant en forêt comme un animal très dangereux, en raison de ses dimensions mais aussi parce qu'elle peut ruer et utiliser ses cornes. 
La vache à laquelle on peut s'identifier, selon les guerriers du clan posóajá, est celle qui est de couleur noire, couleur de la guerre et de la mort.

Les guerriers appartenant au clan jnúminí (dernier du point de vue démographique, social et mythique) disposent, eux aussi, d'une double identification aux edópasade qui leur appartiennent. Un Jnuúminí peut s'orienter vers la foudre (tudasí), car personne ne peut l'éviter. Il peut aussi s'identifier directement à la mort (pitoingai).

Presque toutes les déclarations de courage des guerriers ayorés impliquent, de manière plus ou moins directe, une assimilation au feu. Le recours à cet élément est d'autant plus significatif qu'un des personnages de leurs mythes, Susmaningai, personnifie le courage, non seulement parce qu'il a été le premier à allumer le foyer mais aussi parce qu'il a présidé, au temps des origines, à une preuve ordalique du feu visant à distinguer les êtres courageux, aptes à la guerre et à la chefferie, de ceux qui, n'ayant pas cette qualité, sont considérés comme inutiles (le terme ayoré uusé mélange la notion de stupidité avec celle de folie) ${ }^{22}$. Un des animaux sortis indemnes de ce passage par le feu est quiaquia, auquel, comme nous l'avons vu, le guerrier picaneai s'assimile. Grand tueur pendant qu'il était encore Ayoré, la viande de cet oiseau est strictement interdite et ses plumes sont réservées au chamane. Le mythe de Susmaningai montre aussi la valeur symbolique de la tête et des cheveux. Ces derniers sont l'objet d'une attention particulière des guerriers ayorés : ils les attachent avec une ficelle de cáaguatá de manière à former une sorte de petite queue rigide qui se mêle aux plumes et aux nattes de plumes (potayé) de perroquet attachées derrière le bandeau frontal en cuir.

Le chugui est parfois intégré dans une cérémonie plus ample, le namacade, qui a lieu le plus souvent en période d'abondance pendant la saison des pluies. Cette cérémonie, qui sert surtout à préparer la guerre contre les Blancs, prévoit, entre autres, des jeux rituels entre groupes voisins et entre individus appariés (des jeux d'agilité par exemple) et la consommation de repas communs au sein de clans apparentés. Cette cérémonie confirme le découpage du champ social impliqué par la guerre ainsi que les valeurs symboliques qui lui sont associées. Lors du namacade, pratiqué parmi les Ayorés du Paraguay, surtout par les Totobiégosode, le chugui se fait collectivement autour d'un cactus haut et droit qui est abattu et érigé au centre du campement dès qu'il est bien sec. Le chugui est suivi, d'une part, par des pas cadencés autour du cactus que les guerriers exécutent peints mais sans armes, d'autre part, par la simulation d'attaques de la part de guerriers ennemis qui font irruption dans l'espace rituel cherchant à s'approprier des objets qui composent le trésor symbolique attaché à l'arbre : ornements de plumes, instruments de musique, etc. ${ }^{23}$. Peu après, ou bien le lendemain, les guerriers laissent la place aux jeunes des deux sexes restés au campement, qui accomplissent, le visage peint, la même danse rituelle autour du cactus. Cette cérémonie peut aussi se répéter les jours suivants dans sa fonction propitiatoire, pendant que les guerriers sont déjà engagés dans l'action de guerre. Mais, avant de partir, les guerriers qui 
savent le faire chantent aussi en s'accompagnant d'un grelot (paacá). D'abord les peane, c'est-à-dire des chants sans paroles très diffīiles à exécuter et qui appartiennent à cinq seulement des sept clans, ensuite des chants ordinaires, puisque au fond ce n'est que le simple fait de chanter qui est important, ou bien les cingóangane, des chants qui commencent souvent avec la formule canonique « je dois me lever $»$.

Par ces derniers chants, on s'adresse directement à l'ennemi tout en déclarant, comme dans le chugui, ne pas avoir peur et posséder les mêmes capacités guerrières que lui. Le symbole clanique du chef le plus prestigieux parmi ceux qui organisent la guerre est significativement gravé dans le cactus qui reste au centre du namaccade. Fichermann (1988) nous rapporte que, chez les Ayorés boliviens, tous les symboles claniques sont gravés.

Cet élément est particulièrement important car il montre, avec les assimilations aux edópasade proposées lors du chugui ou avec l'exécution des peane, une des logiques profondes qui réglementent la guerre, surtout intratribale. En effet, sa préparation est centrée sur l'exaltation du clan, qui est à la fois principe identitaire et organisation sociale. Que l'on pense, pour en souligner l'importance, à l'alliance matrimoniale, structurée sur un principe exoclanique qui n'empêche pourtant pas, comme nous venons de le découvrir, une préférence pour une forme de " mariage au plus proche » défini, lui aussi, par l'appartenance à un clan. Que l'on pense aussi à la solidarité clanique, qui oblige à l'aide réciproque des parents de clans, surtout en l'absence de parents généalogiquement proches, ou à l'amitié spéciale (yacotei) qui lie les clans deux à deux ${ }^{24}$ et qui est à la base des échanges cérémoniels. Ces derniers se font, comme on l'a déjà dit, lors de la cérémonie appelée namacade, ou bien, lors d'une autre cérémonie appelée tabóidí, au retour de l'expédition guerrière - échange de miel contre viande par exemple, dont les femmes restent exclues et qui peut servir aussi pour purifier les choses pillées aux Blancs.

La "négation de l'humanité dans l'autre », pour reprendre la formule de Françoise Héritier (1996), est exprimée par les Ayorés d'une manière double. D'une part ils coupent la tête de leurs ennemis pour empêcher qu'on leur donne une sépulture, d'autre part ils ne tiennent pas compte de l'appartenance clanique qui, obligeant à reconnaître le même dans l'autre, en empêcherait la mise à mort. Á l'entre-soi de la consanguinité, du territoire et du genre (Héritier 1999, pp. 324-325), on pourrait ajouter ainsi celui de l'appartenance clanique. Celle-ci se module en effet sur cet entre-soi primaire qui est la consanguinité et en constitue un aspect complémentaire indispensable.

Or, paradoxalement, dans les récits de guerre on ne fait aucune référence au clan des ennemis tués, alors qu'on en rappelle parfois soigneusement les noms. En effet, il n'y a que la solidarité clanique qui est suspendue pendant la guerre, tandis que son exaltation est faite de la même manière par les groupes ayorés ennemis qui partagent aussi - avec des différences insignifiantes - la langue, la culture 
matérielle, les coutumes ou les mythes. Quoiqu'ils ne soient pas confiés à des mécanismes élémentaires, en temps de paix, les mariages entre groupes différents peuvent se faire.

\section{L'AUTRE IMAGE}

La logique de la guerre chez les Ayorés peut se comprendre plus à fond par l'analyse du páagápidí, qui suit l'événement meurtrier. Tout de suite après avoir tué, par exemple près du campement qui a servi de dernière base d'appui avant l'attaque, ou, si la situation ne le permet pas, quelques jours après (mais jamais dans le campement et toujours en l'orientant vers l'est de celui-ci), les Ayorés accomplissent un rituel qui doit les libérer des conséquences néfastes qui accompagnent l'acte meurtrier.

Après avoir nettoyé quelques mètres carrés de forêt, chaque guerrier trace sur le sol un dessin qui représente de façon stylisée une figure humaine : le corps est un rectangle de $30 \mathrm{~cm}$ sur un mètre, chaque membre inférieur et supérieur est représenté par deux ou trois lignes tracées à partir des quatre angles de ce rectangle ; la tête enfin est un petit carré ou cercle qui est juxtaposé à l'un des deux côtés courts du rectangle ou bien elle est figurée en traçant une séparation interne d'un côté du rectangle. Les mains et les pieds (ou plutôt bras et jambes) peuvent manquer, il arrive aussi que des petites variations puissent apparaître dans les dimensions selon qu'il s'agit d'un homme, d'une femme ou d'un enfant. Si un guerrier a tué plusieurs personnes, il peut les cumuler dans un seul páagápidí, qui est en tout cas la preuve du meurtre. Le mort « appartient » en effet à celui qui lui a infligé la blessure mortelle. L'attribution du mort lors de la bataille est tellement importante qu'elle peut même donner lieu à des disputes avec le chef. Signalons enfin que, à la différence de certains Ayorés boliviens ${ }^{25}$, ceux du Paraguay ne semblent pas toujours orienter le dessin appelé páagápidi ainsi que les armes dans une direction particulière, et qu'ils ne font pas de páagápidi pour un jaguar; de plus, les armes qui ont tué ce dernier ne sont pas considérées puyác, et cela bien qu'on lui coupe souvent la tête et qu'on lui accorde le statut de personne en le rangeant parmi les morts dont on tient soigneusement la comptabilité.

Le dessin représente l'ennemi, autour duquel le guerrier tourne une ou plusieurs fois en s'arrêtant surtout à la tête. À celle-ci il réserve un traitement particulier car il l'écrase avec les pieds et avec les mains, qui simulent l'empoignade d'un bâton (Figure 7). En même temps, il lance des cris par lesquels il s'adresse encore une fois directement à son adversaire, lui rappelant qu'il l'a tué, ce qui fait pendant aux cris de défi lancés lors de la préparation de la guerre et qui singularise à nouveau l'ennemi par rapport à une pratique de la guerre poussant à frapper dans le tas. Cette singularisation apparaît d'autant plus paradoxale que les victimes sont le plus souvent des femmes et des enfants que le guerrier ayoré comptabilise tous comme des personnes. 

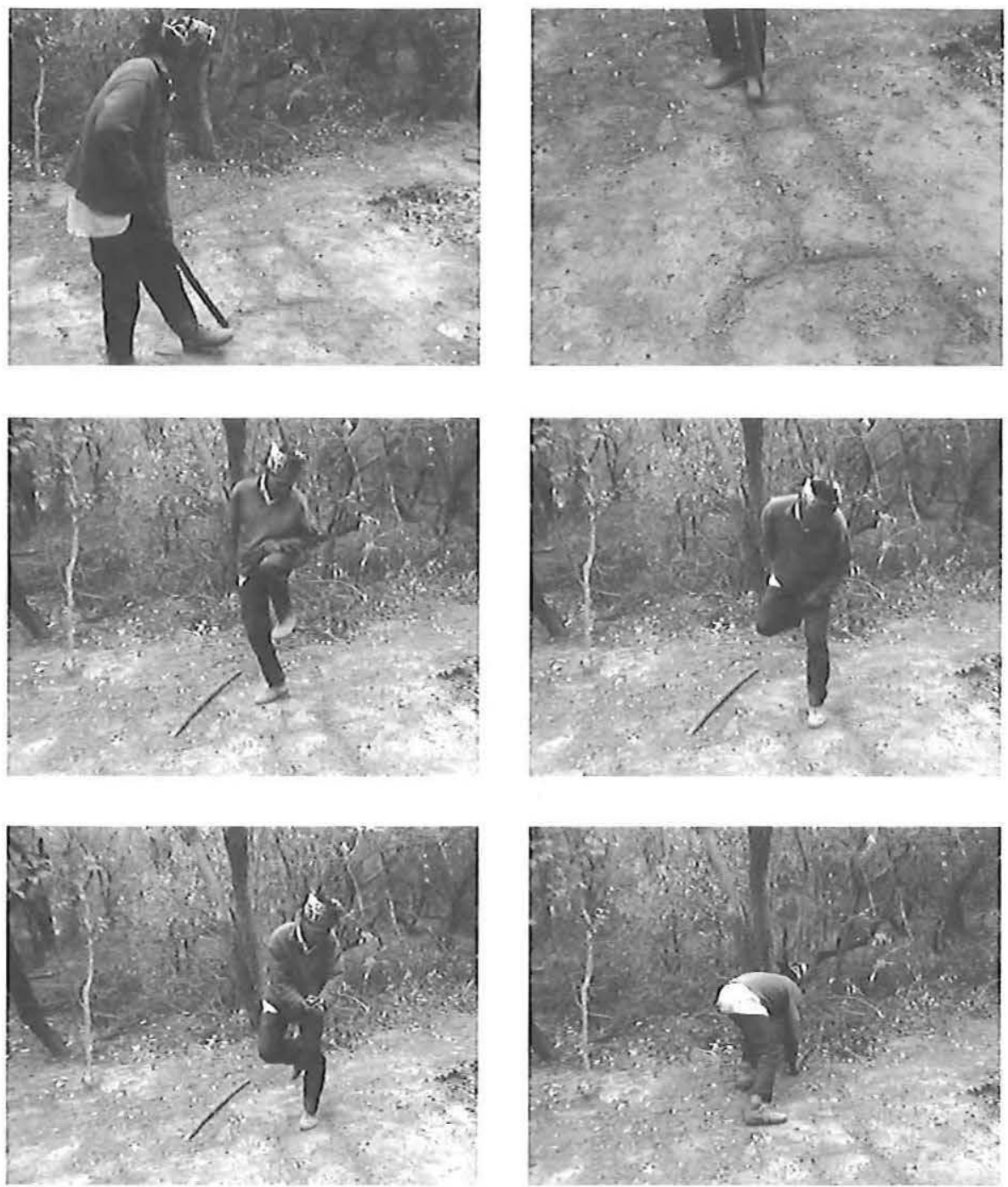

FIG. 7. - Campo Loro, páagápidi, mise à mort du double de l'ennemi (clichés D'Onofrio 2000. Lecture de gauche à droite)

Ce rituel a été justement interprété comme une intervention sur l'oégaté des morts, c'est-à-dire sur une sorte d'âme externe que le rectangle représenterait. Nous préférons intégrer à la définition plus précise qu'en donne Sebag (1965, p. 10) de « double psychique qui peut quitter le corps et survivra à la disparition de celui-ci », celle que les Ayorés eux-mêmes donnent de l'oégaté comme « autre 
image ${ }^{26}$. Oégaté est un mot composé de oé (image, ombre) et gaté (autre, additionnel). Les deux dictionnaires que nous avons déjà cités traduisent le mot entier comme esprit (du mort), apparition, idole mais aussi simplement image. Le caractère de ce double se précise encore plus comme centré sur la tête, car gaté signifie également au-dessus, en haut. Le rituel du páagápidi vise à effacer les fonctions qui reviennent normalement à l'oégaté après la mort d'un individu. Entrée dans le monde chtonien des morts (jnaopié) à travers la sépulture du corps qui lui servait de support mais dont elle est maintenant à jamais détachée, l'oégaté continue à vivre jusqu'à ce qu'elle ne meure à nouveau. Elle pourra donc ressortir sur terre (eami) ou elle continuera sa vie après la mort au travers d'une double incarnation. D'abord, elle trouvera un réceptacle dans un des éléments de l'univers qui forment la propriété symbolique de chaque clan (ce sont donc les edópasade) ; ensuite, après la mort de cet élément (mort spontanée ou provoquée par l'homme s'il s'agit d'un animal ou d'une plante), l'oégaté se déplacera dans la région supérieure (gaté) en attendant de s'incarner dans un nouveau-né. Ce double psychique immortel qu'est l'oégaté et dont se sert particulièrement le chamane ayoré (voir Sebag 1965) parcourt ainsi dans toute leur verticalité les différents mondes reconnus par les Ayorés, en assurant la continuité entre les éléments de la nature qui furent autrefois eux aussi des Ayorés et les nouveau-nés. Par l'intermédiaire de son double psychique, un Ayoré porte donc sur lui en permanence la trace des edópasade qui appartiennent à son clan. La mise à mort de l'oégaté de l'ennemi tué vise vraisemblablement à couper ses liens avec la nature et avec les autres hommes de son même clan dans lequel son oégaté se serait normalement incarné.

Il est significatif que l'oégaté du mort soit représenté dans le rituel par une figure humaine, un double qui permet, comme on l'a vu, de reproduire symboliquement, " comme dans un miroir ", la séparation de la tête du corps. Comme nous l'avons déjà dit, celle-ci est la préoccupation la plus importante d'un guerrier face à l'ennemi tué : lui couper la tête et, quand on peut, la prendre par les cheveux et la joindre aux autres à quelques centaines de mètres du campement ennemi, pour donner aux guerriers de son propre groupe la preuve que l'on a tué et pour que cela serve d'avertissement. En coupant la tête on empêche de donner sépulture au cadavre, qui deviendra ainsi un repas pour les bêtes sauvages.

Aucune explication ne nous a été donnée sur cette prohibition d'enterrement, alors qu'il est souligné avec une certaine complaisance que le cadavre de l'ennemi sera mangé par les fauves. Les Ayorés stigmatisent la coutume d'autres Indiens d'abandonner en forêt les gens très âgés ou qui, après un accident, ne sont plus autonomes au sein du groupe. N'ayant généralement pas le courage de les tuer et pour empêcher justement qu'ils deviennent la proie des fauves, les Ayorés préfèrent les enterrer vivants (eux-mêmes peuvent le demander), ce qui montre combien est " relative » la frontière de ce que nous qualifions parfois indifféremment d'actes sauvages. D'ailleurs, dans le monde des morts, ceux qui ont demandé à 
être enterrés vivants aussi bien que les morts en bataille auront un corps en bonne santé.

Quoique les Ayorés n'en aient pas conscience, c'est pour marquer les effets du démembrement du corps de l'ennemi que le guerrier évite soigneusement, lors du páagápidi, d'entrer à l'intérieur du rectangle, alors qu'il en piétinera plusieurs fois la tête. L'importance de celle-ci a été soulignée par Fischermann (1988) qui rapporte la coutume selon laquelle des Ayorés boliviens placent là où se trouve la tête une petite tortue, grande assassine dans le mythe comme on l'a déjà dit, et qui symbolise à la fois « une affaire de tête » et « le sang ». À ce propos, un lien serait aussi à approfondir entre, d'un côté, l'effacement symbolique du regard de l'ennemi ${ }^{27}$ qui pourrait, comme nous l'ont signalé quelques Ayorés, perdurer après la mort et, d'un autre côté, la mort sociale provoquée par l'inceste (aussi bien généalogique que clanique). Celui-ci s'exprime en ayoré par un terme composé qui renvoie lui aussi à la sphère du regard, à la prohibition de regarder, de choisir à l'intérieur : tagúdedó qui signifie littéralement « il mange ses propres yeux », de agú, " manger », qui signifie aussi " faire l'amour » (tagú c'est la troisième personne du singulier) et edó qui signifie « œil » (dedó : « ses yeux »).

Le regard apparaît donc comme un élément de singularisation aussi bien qu'un facteur d'identité ${ }^{28}$, ce qui est signalé par deux aspects très significatifs de la cérémonie annuelle de l'engoulevent. Lors de cette cérémonie, que l'on peut considérer comme le dispositif symbolique par lequel toutes les valeurs et tous les rythmes de la culture ayoré s'expriment, les hommes et les femmes ne peuvent pas se regarder, alors qu'il est possible pour eux de se parler. Le tabou, entre conjoints et aussi entre amants, intervient justement avant que leur conjonction ne soit possible, une conjonction qui est l'acte conclusif du temps cérémoniel. Les ressources de la vue sont présentes aussi dans le fait d'ériger, toujours à la fin de la cérémonie, un piquet avec les emblèmes claniques, pour qu'Asoojná (l'engoulevent) puisse « voir » qu'en son honneur tous les actes rituels ont bien été accomplis.

Une autre correspondance entre le páagápidi et la cérémonie de l'oiseau est à signaler. La position que le guerrier prend avant d'aplatir symboliquement le double de l'ennemi tué est la même que celle qu'il prend le jour d'Asoojná après les invocations prononcées contre des ennemis : des jaguars, des métis ou bien d'autres Ayorés. Cette position, dont il est dit qu'elle fait éclater au mieux la rage, prévoit que l'on se courbe en rythme en tenant le poing droit derrière le dos, alors que l'avant-bras gauche est appuyé solidement sur la cuisse. L'avant-bras a une haute valeur symbolique. Pendant la guerre, il est souvent rendu visible, d'un côté, par un brassard porté au-dessus du coude et, de l'autre côté, par l'absence de peinture sur les mains. Le guerrier y trace parfois un signe en forme de croix. L'avant-bras a un caractère ambivalent : il représente pour les Ayorés l'une des parties du corps contaminée par le sang de l'ennemi, mais il est aussi un « réservoir de puissance » (Bormida 1978-1979) qui peut être tournée (parfois avec la 
lance à la main) vers la tempête pour l'arrêter ; la contamination fait respecter un guerrier au sein de son groupe et provoque la terreur chez les ennemis. Signalons comme élément de comparaison que, chez les Aztèques (Sahagún 1982, pp. 380-381), l'avant-bras gauche des ennemis ainsi que celui des femmes mortes en couches était très recherché par les guerriers, pour la force protectrice ou offensive qu'il était censé receler. L'avant-bras des ennemis était également récupéré par les guerriers Txicao (Menget 2000) ${ }^{29}$. Les Yagua (Chaumeil 2001, 2002) récupéraient les humérus des ennemis tués pour les transformer en flûtes qu'ils utilisaient pour terrasser d'autres ennemis. En effet, il s'agit d'une intervention de type chamanique (le souffle qui vient de l'intérieur) qui a, par inversion, des correspondances avec l'utilisation de son propre avant-bras chez les Ayorés.

À côté du traitement symbolique du corps de l'ennemi, une intervention sur les armes meurtrières est nécessaire car, selon la croyance, le sang de l'ennemi les a rendues inutilisables à cause de leur impureté. Si quelqu'un d'autre entre en contact avec ces armes, il peut rester paralysé, donc voué à la mort, exactement comme ceux qui n'ont pas exécuté les prescriptions rituelles de la fête d'Asoojná. Pour conjurer ce danger, deux possibilités sont offertes au guerrier ayoré : soit il les abandonne en les enfouissant sous des feuilles mortes à côté du dessin, soit il les récupère en les purifiant rituellement par le feu. La récupération des armes qui ont tué n'est justifiée que par l'affection du guerrier à leur égard : " s'ils aiment leurs armes $[\ldots]$ ", comme disent les guerriers ayorés.

Dans le premier cas, le geste accompli par le guerrier est identique à celui réalisé par une personne âgée lors de la cérémonie de l'oiseau. À cette occasion, il utilise un bâton de najnui ${ }^{30}$ et tue symboliquement les chasseurs qui rentrent un par un de la forêt en les frappant sur le fessier. Or ce bâton est passé à chaque fois sous des feuilles mortes avant de servir pour le chasseur suivant. À la fin de la cérémonie, il est jeté dans la forêt.

Si le guerrier veut récupérer son arme, il peut tout simplement la tourner sur un feu qu'il a allumé à côté du dessin (et il fait de même avec les parties de son corps que le sang de l'ennemi a tachées ${ }^{31}$ ) ou bien avoir recours à un rituel un peu plus complexe qui se fond avec l'écrasement symbolique de l'ennemi que nous venons de décrire. Ce rituel prévoit que l'on plante les armes meurtrières (appelées guitade) aux pieds du páagápidi, en les enfonçant obliquement pour qu'elles se croisent. Sous l'endroit où les armes se croisent, on pose une petite braise ainsi qu'un petit morceau de n'importe quelle viande qui est destiné à recevoir le sang qui coule symboliquement d'elle (Figure 8). Au terme de ce parcours à rebours, qui réunit le sang à la chair tout en assimilant implicitement la chasse à l'activité meurtrière, les armes qui ont tué peuvent être gardées par le guerrier auquel elles appartiennent, quoique leur usage connaisse, à partir de ce moment, des limitations concernant surtout la femme du guerrier. Par exemple, elle ne pourra plus manger de la viande de pécari qui aura été tué par cette arme ${ }^{32}$. Il faut avoir fait le dessin pour pouvoir coucher avec sa femme, faute de quoi on risque des 


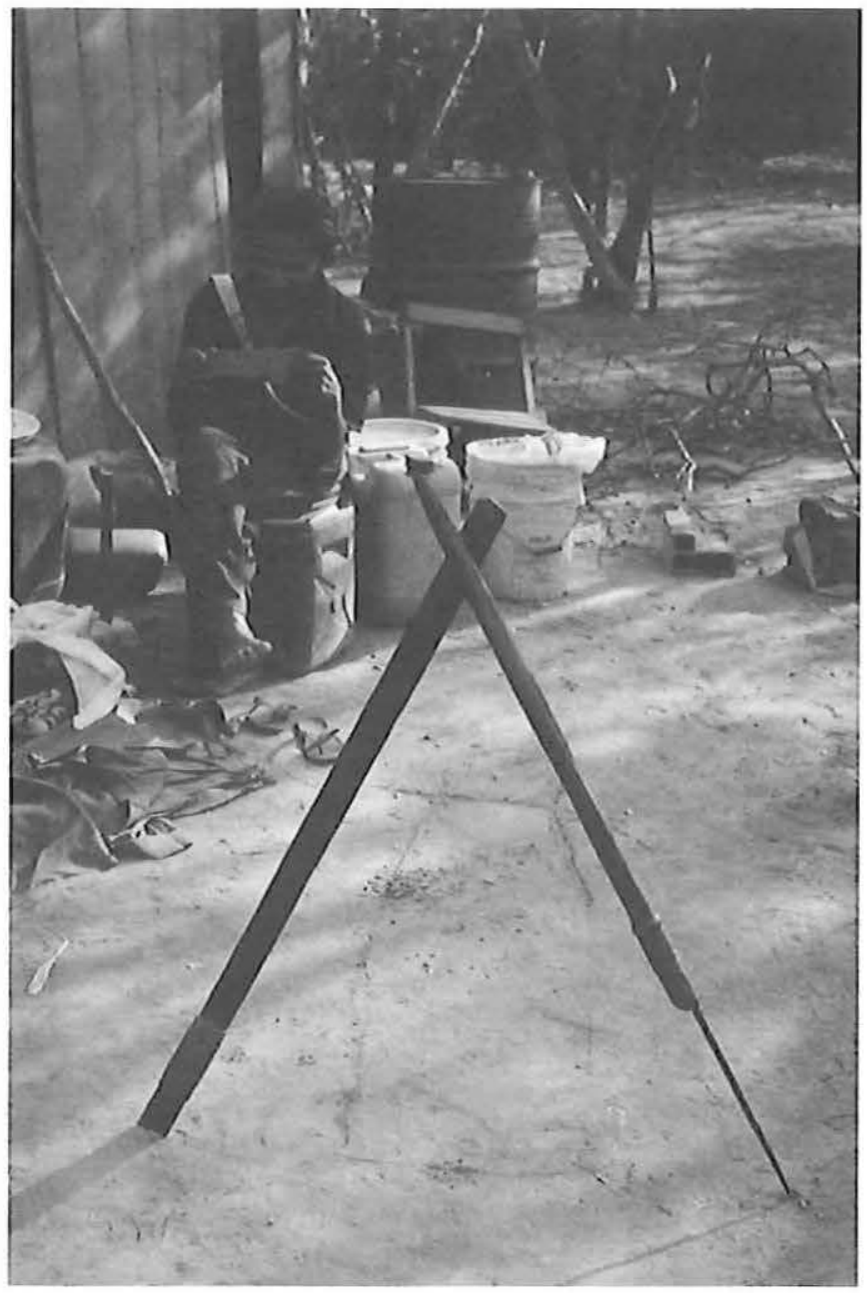

FIG. 8. - Túnucojnai, variante du páagápidi (cliché D’Onofrio 2001)

maladies. Le lieu où le páagápidi a été fait sera complètement abandonné. Une attitude très différente se manifeste à l'égard des armes prises à l'ennemi. N'étant pas considérées comme puyác, celles-ci ne nécessitent aucune intervention particulière pour pouvoir être utilisées. Alors que les propres armes sont associées à la mort, et à la mort symbolique qu'est la paralysie (qu'elles peuvent provoquer simplement en entrant en contact avec quelqu'un), on n'inscrit pas les armes de l'ennemi dans un horizon rituel parce qu'on suppose qu'elles ont déjà été purifiées 
après avoir donné la mort. Mais cette absence de ritualisation montre aussi que la culture ayoré tend à refouler la puissance d'autrui. C'est peut-être pour cela que l'on ne se réfère généralement pas aux défaites et que les attaques meurtrières que l'on a soi-même subies ne deviennent pas l'objet de récit.

Certains de nos informateurs n'ont accordé d'autre signification au fait de tuer des ennemis que celle de la puissance croissante que le guerrier peut y gagner. Celui-ci définit son identité par rapport au territoire où il a l'habitude de nomadiser et à l'intérieur duquel les campements permanents ou temporaires sont établis. La dénomination de chaque groupe ayoré relève, au fond, des caractéristiques d'un territoire reconnu par les autres, ce qui n'a pas empêché les fusions (Figure 9). Le terme guidáigosode, qui signifie « les gens qui vivent dans les campements (durables) ", est appliqué par exemple par les Ayorés de Bolivie à tous ceux du Sud (qui vivent au-delà des salines dans le territoire paraguayen). L'entité ainsi dénommée s'est formée suite à un processus d'unification qui est intervenu dans les années de leur réduction à la civilisation des Blancs. Les divisions n'avaient pourtant pas empêché les Guidáigosode de s'allier de temps en temps avec leurs ennemis acharnés, les Gaáigosode, pour faire la guerre aux Ayorés du Nord et, depuis quelques décennies (après leur fusion), pour faire la guerre aux Totobiégosode... S'ils ne sont pas des alliés, les autres groupes ayorés sont toujours considérés comme des ennemis potentiels ou bien comme des gens avec qui le mariage est possible à condition qu'ils quittent leur groupe ou qu'ils soient globalement assimilés.

La guerre intra-ethnique chez les Ayorés confirme ce mode d'incorporation de l'altérité qu'est la prédation, contrainte fondamentale de l'existence animale dont le modèle appliqué à l'Amazonie (Viveiros de Castro 1992, 1993 ; Overing 1993) a supplanté les interprétations traditionnelles de type écologique (Harris 1984) ou socio-biologique (Chagnon 1988). Le mode prédateur de rapport à l'autre n'exclut cependant pas que la relation de guerre puisse se convertir, le cas échéant, en relation d'échanges de tout genre.

En général, nous pouvons affirmer que les différents groupes ayorés incarnent à tour de rôle les uns envers les autres les statuts d'alliés et d'ennemis qui, eux, demeurent constants. L'espace social ayoré est donc caractérisé par le cumul des deux types de guerres conventionnelles qui ont été reconnus dans les sociétés traditionnelles (Descola et Izard 1991) : à savoir, la guerre comme substitut de transactions pacifiques (d'échanges commerciaux et/ou matrimoniaux) ou comme pratique visant à assurer la reproduction symbolique de sa propre identité au sein du cosmos. Chez les Ayorés, la guerre satisfait donc à la fois, d'une part à l'exigence de "se procurer, chez un ennemi très spécifiquement défini, des principes ou des substances nécessaires soit à la perpétuation de l'identité du groupe [...], soit au maintien du bon ordre du monde dans son ensemble » (ibid., p. 314), d'autre part à l'exigence de pouvoir inscrire le corps à corps avec les autres groupes ayorés dans des horizons opposés (pacifiques ou conflic- 


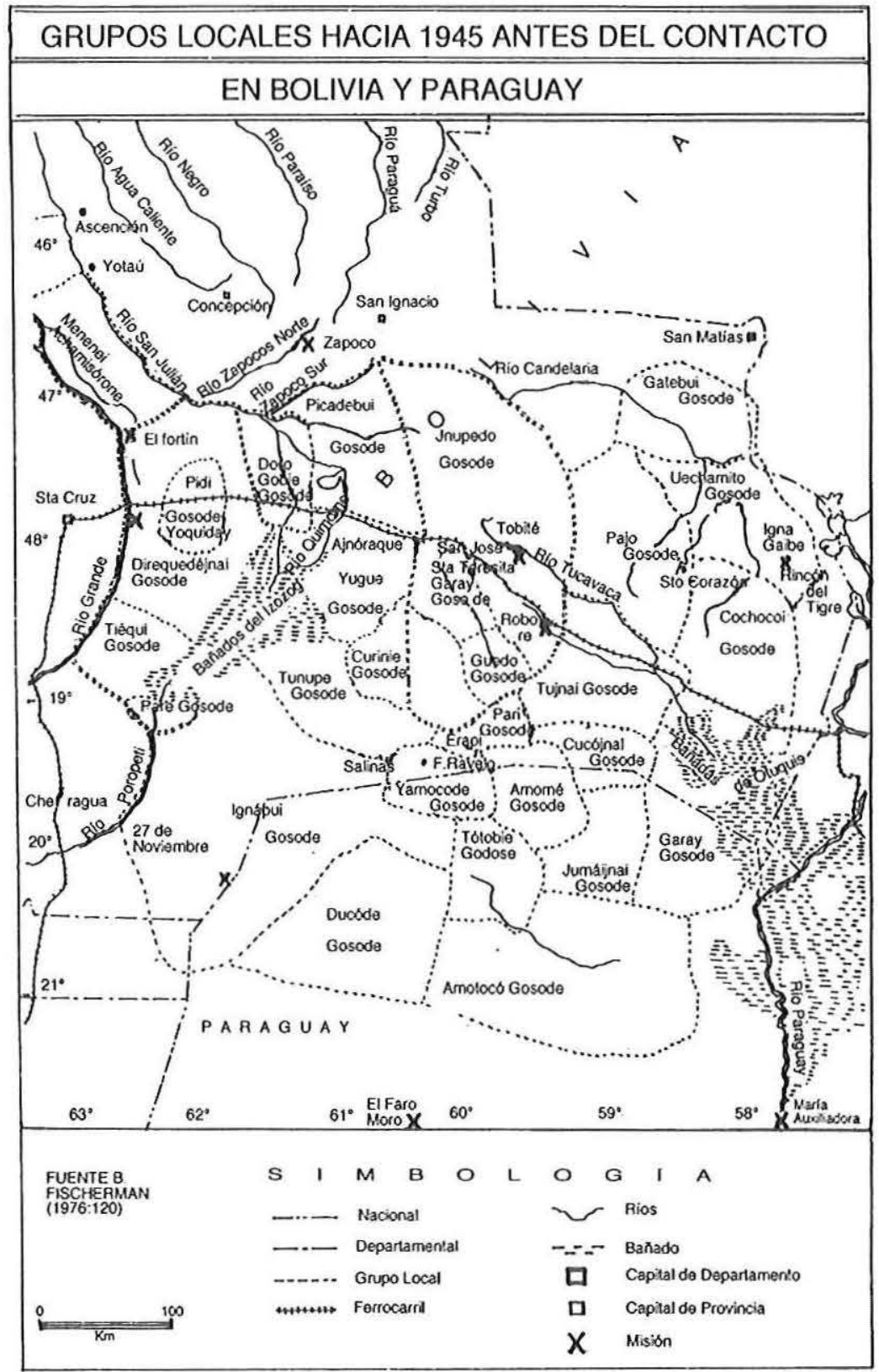

FIG. 9. - Localisation des groupes ayorés (Bartolomé 2000) 
tuels) mais en même temps réciproquement convertibles. La dynamique interne à la société ayoré confirme ainsi l'hypothèse d'un lien étroit entre la sociabilité et la violence, récemment formulée par Claude Lévi-Strauss (2000a, 2000b). En prenant comme modèle la vie des amibes, êtres monocellulaires dont on a étudié soigneusement le passage de l'isolement à la vie en société, Lévi-Strauss montre que l'antinomie entre l'" animalisation » et la « divinisation » de l'homme (homo homini lupus, homo homini deus) n'est qu'une question de degré. La sociabilité apparaît en d'autres termes comme "limite inférieure de la prédation », étant donné que les mêmes substances qui relient les proies aux prédateurs sont capables de les attirer les unes vers les autres pour les réunir en société.

\section{LA SUBSTANCE}

Si on leur pose la question, les Ayorés ne réalisent pas immédiatement l'idée d'un transfert de substance de l'ennemi à celui qui a tué. Cependant, si nous analysons les métamorphoses du temps des origines que les guerriers ayorés actualisent tout au long de leur vie, dans les cérémonies annuelles aussi bien qu'en racontant des mythes ou à l'occasion d'une guerre, nous découvrons un transfert qui est même double en ce qu'il permet de mettre en correspondance l'horizon du mythe avec la sphère des pratiques symboliques et qui est aussi un aspect important de l'organisation sociale, surtout dans la guerre intratribale. Le sang de l'ennemi, par exemple, révèle tout son caractère ambivalent en ce qu'il est perçu, d'une part comme substance souillante dont il faut nettoyer par le feu les parties du corps et les armes qui ont été tachées, d'autre part comme nourriture privilégiée par les animaux mythiques auxquels les Ayorés s'assimilent à travers leurs gestes et leurs mots, surtout rituels.

Comme autre preuve du désir de se libérer rapidement du sang souillant de l'ennemi, signalons l'utilisation après la tuerie de la pota, la flûte, symbole elle aussi de la tête, à l'intérieur de laquelle il faut souffler pour éliminer les traces sanglantes de l'ennemi. Si le son ne sort pas bien, c'est le signe que le sang de l'ennemi y est encore enfermé.

En revanche, c'est en absorbant symboliquement le sang de l'ennemi que l'on devient puyác, c'est-à-dire aussi puissant que les oiseaux dont il est dit dans les mythes qu'ils ont tué lorsqu'ils étaient encore Ayorés et dont on ne peut utiliser les plumes qu'à partir du moment où on a tué. Ces ancêtres oiseaux sont les Ayorés qui, ayant tué le puopié, la puissance contenue dans le tabac, ont pu s'adonner à l'activité chamanique. L'aspect positif de la contamination par le sang traduit bien l'ambivalence de la notion de puyác. Le fait d'avoir absorbé la substance sanguine de l'ennemi demande des actes de purification rituelle mais il augmente aussi la puissance du tueur autant qu'ill'immunise. Le caractère périlleux du contact avec le tueur (qu'il soit chef ou chamane) s'étend fondamentalement à 
toutes les choses qui font partie de son espace existentiel et se manifeste sur les enfants, les femmes et les adultes qui n'ont jamais tué.

On peut noter une autre homologie de substance. Le miel, quintessence de la nourriture crue, symbolise pour les Ayorés le sang. Sa consommation par les femmes est assujettie pour cela à de nombreuses limitations, alors que les hommes le consomment, surtout dans les occasions cérémonielles, comme s'il s'agissait d'un substitut du sang de l'ennemi. En mangeant le miel lors de la fête de «l'oiseau » (l'engoulevent), les Ayorés de Bolivie disent littéralement yacobu catai iyoi ou bien yacobu coñone iyoi [" nous mangeons rapidement le sang du jaguar » ou bien « des Blancs »]. L'association entre le sang et le miel relève d'une idéologie qui assimile pour beaucoup d'aspects l'activité de chasse et de récolte du miel à la guerre : lorsqu'on va chercher du miel, on peut se peindre de la même façon que si on allait à la guerre (Susnik 1990, pp. 13-14), les récits de guerre montrent une relation très étroite entre ces activités. L'incipit d'un récit que nous avons recueilli à Campo Loro dit par exemple: «Quelques jeunes et moi, le lendemain nous devions aller chercher du miel ou bien chasser des animaux ou bien des Ayorés. Nous allions, nous allions, nous allions en ce moment ".

Nous ne savons pas si les Ayorés boliviens font allusion aussi au sang des ennemis ayorés ; cependant, ces derniers se retrouvent à côté des Blancs et des jaguars, dans les requêtes « criées » à Asoojná par les Ayorés paraguayens lors de la cérémonie annuelle. En outre, le rituel de récupération des armes par le feu et de réintégration du sang qui coule sur le morceau de viande que l'on place sous elles a surtout lieu lorsque l'ennemi, sur la tête duquel on est symboliquement intervenu, est lui aussi un Ayoré.

Ce double mouvement d'incorporation et de répulsion est renforcé par l'attitude rituelle que l'on a à l'égard du corps de l'ennemi, car on lui accorde sur le plan symbolique ce qu'on lui nie dans la pratique, c'est-à-dire la possibilité d'une sépulture. Le rituel du páagápidí se conclut en effet avec la transformation du double de l'ennemi en sépulture lorsqu'une poignée de terre est jetée sur le dessin. Par ailleurs, l'aplatissement au sol qui empêche l'oégaté du mort de bouger est, lui aussi, une sorte d'enterrement symbolique.

Nous pouvons en conclure que la construction de l'identité ne passe pas seulement par la dissolution substantielle d'autrui mais aussi, et en même temps, par son incorporation symbolique. Dans le cas des Ayorés, cela s'explique par l'exigence de convertir l'ennemi interne en allié : c'est ce qui est souvent arrivé, comme on l'a déjà dit, entre les groupes des Guidáigosode et des Totobiégosodemais aussi entre ces groupes du Sud et ceux du Nord (les Ayorés boliviens). Nous pouvons ainsi placer dans un nouveau cadre le débat autour des rapports entre guerre et échange. Dans le contexte de cet article, on ne retiendra que les positions de Pierre Clastres (1977) car elles ont soulevé sur la question une discussion qui est toujours d'actualité ${ }^{33}$. Clastres soutient que la guerre et l'échange ne se situent pas sur le même plan. L'idée que l'on ne puisse pas penser la société 
primitive en dehors de la violence est mal formulée, car la tendance de l'homme en société est de canaliser l'agressivité et les tensions vers la guerre en l'absence d'autres mécanismes ; les sociétés amérindiennes elles aussi élaborent différentes stratégies, susceptibles de changer de signe en fonction des rapports entretenus avec l'autre. En effet, chez les Ayorés, l'autre peut en général être transformé en allié, surtout s'il est également Ayoré. La thèse de Clastres selon laquelle la " guerre est une structure de la société primitive et non le résultat d'un échange manqué » ne doit être retenue qu'en partie. D'abord, cette structure est (malheureusement !) un trait de toutes les sociétés humaines. Ensuite, s'il est vrai que la causalité initiale de la guerre peut ne pas être toujours dans l'échange (raté), il n'en est pas moins vrai que l'on peut arriver à l'échange par la guerre. Guerre et échange s'impliquent dans une situation d'exclusion réciproque à un moment donné, comme l'a vu Lévi-Strauss (1943) et comme le confirment les documents ethnographiques. Rien de plus bizarre en effet que de penser à la société primitive comme à une " société contre l'échange » car le fait de vouloir vivre entre soi n'a jamais exclu l'envie de pratiquer, le cas échéant, la voie de l'échange. C'est justement la guerre intra-ethnique qui montre au plus haut degré non seulement l'exclusion mais aussi la possible conversion réciproque entre guerre et échange. Cette convertibilité est à l'origine de certains usages linguistiques et cérémoniels sur lesquels des travaux ethnologiques récents ont attiré l'attention. Philippe Descola (1986a, 1986b, 1993a) et Anne-Christine Taylor $(1985,1994)$ ont notamment signalé chez les Jivaro le traitement du beau-frère comme ennemi dans la chasse au gibier et dans les jeux rituels. Et, dans un cadre comparatiste, Carlos Fausto (2001) observe que les termes indigènes pour vendetta renvoient souvent à des formes d'échange proches de celles de l'échange économique. Cette convertibilité entre guerre et échange permet également de préciser dans quel sens la proposition de Fausto qui considère " la guerre comme échange » peut être accueillie. Nous pensons notamment à la possibilité d'élargir la réflexion à l'échange de substances ${ }^{34}$ qui sous-tend les pratiques de la guerre et qui rejoint la ligne de recherche tracée par Françoise Héritier. Nous pensons aussi aux médiations symboliques entre l'homme et la nature (autant interne qu'externe) qui reprennent leur place dans le débat anthropologique contemporain avec les travaux de Descola (voir le propos de sa leçon inaugurale au Collège de France 2001).

En ce qui concerne les Ayorés, ces médiations et la valeur ambivalente du sang dans la relation à l'ennemi, surtout interne, sont clairement représentées dans le rituel. En éliminant de soi, de son corps ainsi que de ses armes, les traces souillantes de la substance de l'autre, le guerrier ayoré fait en sorte que l'incorporation symbolique de cette même substance lui permette de réactiver le circuit de l'alliance. En effet, si les contingences de la dynamique politique le permettent, l'autre peut redevenir une partie de soi en ce qu'il est possible de voir en lui non pas le membre d'une totalité indistincte, mais d'une totalité qui s'articule socia- 
lement de la même manière que son propre groupe. Entre les deux pôles de la substance repoussée et de la substance incorporée, le sang qui tache et celui qui nourrit, il y a place pour rétablir, dans les relations intratribales, ce principe structurel d'organisation de la vie sociale qui est l'appartenance clanique, une sorte de substance mystique que l'on peut considérer à la fois comme autre - car, appartenant à des clans différents, le mariage est possible - et comme identique car l'appartenance au même clan empêche le mariage. Le rituel préfigure donc la possibilité de reprendre entre soi et l'autre le jeu qui structure, de l'intérieur, la société ayoré.*

* Manuscrit reçu en septembre 2002, accepté pour publication en mars 2003.

\section{NOTES}

Remerciements: Les données ethnographiques de cet article résultent d'un terrain de presque un an au total $(1998,1999,2000,2001)$ chez les Ayorés du Paraguay, terrain financé par le rectorat de l'université de Lecce dans le cadre d'un accord avec l'université catholique d'Asunción. Je tiens ici à remercier le directeur du CEADUC, Dr. José Zanardini, et ses collaborateurs pour avoir facilité mon séjour au Paraguay. Une première version de ce texte a été présentée en avril 2002 au séminaire de Philippe Descola à l'École des hautes études en sciences sociales ; je le remercie pour la générosité des discussions et des conseils qui ont suivi et pour m'avoir soutenu dans la réorientation de mes études vers l'américanisme. Je veux exprimer également toute ma gratitude à Jean-Pierre Chaumeil, Philippe Erikson, Michel Perrin, Ugo Fabietti, Isabelle Daillant, Pierre Beaucage pour leurs commentaires. Je considère comme un privilège d'avoir eu la possibilité de parler de mes recherches sur les Ayorés à Claude Lévi-Strauss au cours de quelques rencontres inoubliables. Je le remercie pour son appréciation et pour ses encouragements. Enfin, je dédie cet article à la mémoire de Lucien Sebag.

1. Nous empruntons les exemples qui suivent à De Mauro (1970).

2. Nous utilisons pour l'ethnonyme la transcription francisée, tout en signalant que celle-ci est calquée sur la transcription espagnole qui a introduit le -r-, que les Ayorés du Paraguay ne connaissaient pas, et modifié la formation du singulier et du pluriel (originairement Ayowé sing., Ayowéode pl.) : Susnik (1963), dans une enquête menée à l'époque du premier contact, transcrit " ayoweos »; Sebag aussi, dans son cahier de terrain, note Ayubeo à côté de Moro, terme méprisant utilisé par les Paraguayens, qui sont eux dénommés par les Ayorés coñone (" ceux qui ne savent rien »). Si nous adoptons l'hispanisation, c'est à la suite des Ayorés qui sont nés " dans la civilisation »-comme ils le disent eux-mêmes - et qui ont eu une formation scolaire. Il n'empêche que ceux-ci ont tendance à ne pas utiliser ce -r- quand ils s'adressent aux gens plus âgés qui, pour la plus grande partie, ne parlent que quelques mots d'espagnol. Pour les autres mots, tout en adoptant la graphie espagnole (qui ne prévoit qu'exceptionnellement le redoublement des consonnes), nous préférons transcrire selon la prononciation traditionnelle. Nous signalons aussi que les voyelles en fin de mot, sauf indication contraire, n'ont pas d'accent, tandis que les voyelles nasalisées sont précédées par le signe 'jn'.

3. Il est certainement difficile d'appliquer la notion de "clan » aux Indiens des basses terres, d'autant plus que l'on s'est largement interrogé sur la pertinence même de cette notion par rapport à ses significations d'origine (groupe d'unifiliation, association au totémisme, définition territoriale). $\AA$ défaut d'un meilleur terme, nous utilisons cependant celui de clan tout en signalant que le mot ayoré cucheai n'a aucune relation avec un territoire et que les noms des sept clans n'ont pas de signification. Susnik (1963) trouve néanmoins des affinités avec les noms des clans chamacoco qui, eux, ont une 
signification (les deux groupes composent la famille linguistique Zamuco). Une note isolée de Sebag, publiée par Carmen Bernand (1977), fait allusion à une distribution circulaire des sept clans ayorés dans un même campement. Il s'agit sans doute d'une fausse donnée car le mécanisme même de l'alliance matrimoniale implique que tous les clans soient confondus au sein de la même famille élargie.

4. Les Ayorés cultivaient six produits au total : haricots, maïs, pastèque, deux espèces de courge et tabac pour les chamanes (le seul produit qui n'est plus cultivé ni consommé).

5. En privilégiant l'inscription des récits et des pratiques de guerre des Ayorés dans l'horizon mythique et rituel, nous avons laissé de côté la perspective centrée sur le discours telle qu'elle a été élaborée dans des travaux désormais classiques des années 1990. Nous sommes néanmoins très intéressés par quelques-unes des pistes de recherche tracées par ces approches, notamment à propos des formes du parler (voir par exemple Sherzer 1982, 1987 ; Basso et Sherzer 1990) et sur les questions du style que nous voudrions aborder dans un prochain travail en considérant un double registre : d'une part, comme le suggère Urban (1990, p. 107), en insérant le discours « into a plane of analogous social phenomena, such as body painting, hair and clothing styles, ornamentations, postures, and gestures ", d'autre part, en travaillant sur le style personnel des conteurs. Loin d'être perçu comme une déviation individuelle à des normes et des canons socialement établis, ce dernier est apprécié parce qu'il véhicule les contenus sémantiques qui sont dans le système d'attente de la communauté.

6. II s'agit généralement des trois types de lances connues par les Ayorés mais aussi de l'arc et du bâton de défense. De nos jours, toutes sortes de bâtons ou d'instruments de travail en forme de bâton sont utilisées pour le récit.

7. Selon Pallestrini et Perasso, chaque cordon de plumes (potayé), intégré parfois par des becs de toucan, « significa el número de enemigos muertos » (1988, p. 31 ; cit. in Escobar 1993, p. 159). Plus généralement, ce sont surtout les chefs qui mettent des petits objets parmi les plumes pour faire du bruit.

8. Cet oiseau recommençait à chanter vers la pleine lune du mois d'août, quatre lunes après la montée au ciel de l'étoile rouge Guedo caté (Arcturus). Sur le rapport entre mythes et fête d'Asoojná, voir Lévi-Strauss (1985, pp. 63 et sq.)

9. Voir les deux versions de ce mythe recueillies par Pagés Larraya (1973, p. 259) et Bormida (1974, pp. 61-62), ainsi que l'étude d'Idoyaga Molina (1989, pp. 31-37). Voir aussi la traduction anglaise dans le recueil de Wilbert et Simoneau (1988).

10. Ce concept de " sphères d'identité ", que nous empruntons à Philippe Descola (1993b), rend bien l'idée d'une morphologie socio-spatiale qui accorde beaucoup d'importance aux relations entre une multiplicité de centres auxquels l'individu se sent rattaché et les périmètres qui leur correspondent.

11. Nous avons recueilli une quarantaine de textes qui ont été traduits de l'ayoré à l'espagnol par des Ayorés bilingues et de l'espagnol au français par l'auteur, S. D.

12. À l'époque de notre enquête, Paojnai et Iboé venaient d'arriver depuis quelques mois. Il s'agit d'un couple incestueux frère/sœur qui vit actuellement entre Campo Loro et Túnucojnai.

13. Il s'agit d'une des trois espèces de tapirs connues au Paraguay, mais nous ne sommes pas en mesure de préciser laquelle.

14. On pourrait par exemple comparer ce geste d'abord avec ceux des Indiens d'Amérique du Nord, ensuite avec ceux des populations asiatiques, car il n'existe pas d'équivalents dans d'autres groupes des basses terres d'Amérique du Sud. La comparaison serait néanmoins intéressante avec l'impikmartin des Jivaros de la Haute-Amazonie (Descola 1993a, pp. 314-316) qui déclament eux-aussi contre l'ennemi " en sautant d'un pied sur l'autre » ou avec les Korubo brésiliens qui racontent en frappant quelque chose de temps en temps avec un long bâton qu'ils tiennent dans leurs mains (communication personnelle de Philippe Erikson).

15. La récolte d'une plante en forêt est considérée elle aussi comme une mise à mort.

16. D'autres motifs peints sur le visage, avec la couleur rouge extraite d'une petite pierre en la grattant à l'aide de l'eau sur une pierre dure, sont par exemple deux ou trois lignes transversales sur les joues ou des pointes de flèche toujours tournées vers la bouche, un ou deux bandeaux sur le front. Ces motifs prennent des noms différents.

17. Voir les versions recueillies par Bormida (1974, pp. 61-62) ou Mashnshnek (1990). 
18. Le nom de Uejái provoque encore aujourd'hui dans les villages ayorés, plusieurs années après sa mort, un respect considérable.

19. Mariadé est un teknonyme. Chez les Ayorés, les parents prennent le prénom de leur aîné en ajoutant le suffixe -dé pour le père, -daté ou-naté pour la mère. Dans le cas de Mariadé, c'est le prénom imposé par les chrétiens qui sert de base alors que, normalement, le teknonyme se forme à partir d'un prénom ayoré donné par la grand-mère maternelle. Oji est le prénom ayoré de Mariadé, père de María (prénom chrétien donné à sa fille aînée). Sur ces questions, voir D’Onofrio (à paraître).

20. Nous aborderons, dans un autre travail, les aspects pratiques concernant l'organisation de la guerre; indiquons ici seulement que, par respect, on ne convoquait pas à travers des messagers des chefs aussi importants que celui qui était considéré comme le plus prestigieux. Ces derniers auraient appris également la nouvelle et se seraient rendus tous seuls à la réunion.

21. Une liste de ces edópasade, terme qui fait référence aux entités mais aussi aux ancêtres mythiques dont elles sont issues, a été élaborée par Bartolomé (2000, pp. 264-265) sur la base des données recueillies par Fischermann (1976) et Zanardini (1994). Voir aussi Bormida (1978-1979, pp. 53-71).

22. Voir la note 9. Nous avons présenté l'analyse de ce mythe lors de la journée d'études « La guerre en tête " organisée avec A.-C. Taylor le 31 janvier 2003 au Centre G.-A. Haudricourt de Villejuif.

23. Un jeu rituel dont le souvenir est désormais très faible prévoyait que l'on descendit ces objets de l'arbre, surtout les instruments de musique, sans faire aucun bruit.

24. Cette alliance cérémonielle relie en permanence les clans de la manière suivante : ciquenoiposóajá / etácoé-dosapéi / picaneai-cutamuajane. Le septième clan, jnuúminí, reste le plus souvent exclu ou bien il peut s'allier au clan dosapei.

25. Bormida (1975, pp. 105-106) relate des opinions différentes de ses informateurs.

26. Les Ayorés ont un autre double psychique, ayipie, que l'enfant reçoit de son père et qui meurt avec l'individu. Sur la relation entre oégaté et ayipie, voir Sebag (1965).

27. Dans un texte de récit de guerre, recueilli en 1973 à Maria Auxiliadora en haut Paraguay par Braunstein (1976-1977, p. 41), qui en a proposé aussi l'analyse sémantique, le conteur craint d'être aveuglé par la femme de l'homme qui pourrait le tuer pendant l'attaque (déclenchée en réalité contre les Blancs) : " pensé que iba a morir / si uno de ustedes me va a matar, su señora va a clavarme mi ojo » [je pensais que j'allais mourir / si quelqu'un d'entre vous va me tuer, sa femme va clouer mes yeux].

28. Un principe analogue de singularisation a été découvert par Anne-Christine Taylor (1994) dans la « chasse aux visages » des Achuar de l'Équateur.

29. Dans un des récits de guerre que nous avons recueillis à Campo Loro, on coupe l'avant-bras à un ennemi ayoré. Mais ce n'est qu'un cas sur lequel nous n'avons pas pu approfondir l'enquête.

30. Cette espèce végétale, qui n'est pas recensée dans le travail de Schmeda-Hirschmann (1994) sur l'ethnobotanique ayoré, n'est utilisée que dans le contexte rituel de la fète d'Asoojná.

31. Le geste précis consiste à faire semblant d'essuyer, avec ses mains, de haut en bas ses avant-bras ou ses cuisses au-dessus du feu.

32. À ce propos, il est intéressant de signaler que le statut d'ennemi est souvent attribué au pécari dans les cultures amérindiennes. Une autre interdiction concerne le miel récolté au retour d'une expédition guerrière : les femmes restées au campement ne peuvent pas le consommer.

33. À cette question fait référence, d'ailleurs, la synthèse proposée par Fausto $(2000)$ dont la bibliographie est tout à fait récente.

34. De substances et d'énergie, comme l'a montré, parmi d'autres, un travail de Jean-Pierre Chaumeil (1985) sur les Yagua du Pérou. 


\section{BIBLIOGRAPHIE}

Barrios Armindo, Domingo Bulfe y José Zanardini

1995 Ecos de la selva. Ayoreo de Uruode, Colección Biblioteca Paraguaya de Antropología, XXII, Asunción.

BARToLomé Miguel Alberto

2000 El encuentro de la gente y los insensatos. La sedentarización de los cazadores ayoreo en el Paraguay, Instituto Indigenista Interamericano-Centro de Estudios Antropológicos, Universidad Católica, México.

BAsso Ellen y Joel SHERzer, eds.

1990 Las culturas nativas latinoamericanas a través de su discurso, Abya-Yala, Quito.

BERNAND MuÑoz Carmen

1977 Les Ayoré du Chaco septentrional. Étude critique à partir des notes de Lucien Sebag, Mouton, Paris-La Haye.

BormIDA Marcelo

1973-1979 «Ergon y mito. Una hermenéutica de la cultura material de los ayoreo del Chaco boreal ", Scripta Ethnologica, 1973, 1 (I), pp. 9-68 ; 1974, 2 (II), pp. $41-107$; 1975, 1 (III), pp. $73-130 ; 1976,4$ (IV), pp. 29-44; 1978-1979, 5 (V), pp. $6-25 ; 5(\mathrm{VI})$, pp. $21-75$.

BRAUNSTEIN José A.

1976-1977 "Cigabi va a la matanza. Historia de guerra de los ayoreo », Scripta Ethnologica, 4 (II), pp. 32-51.

BRigGs Janet R.

1973 "Ayoré narrative analysis », International Journal of American Linguistics, XXXIX (3), pp. 155-163.

Chagnon Napoleon A.

1988 "Life Histories, Blood Revenge, and Warfare in a Tribal Population ", Science, 239, pp. 985-992.

Chaumeil Jean-Pierre

1985 «Échange d'énergie : guerre, identité et reproduction sociale chez les Yagua de l'Amazonie péruvienne ", Journal de la Société des Américanistes, 71, pp. 143-157.

2001 "Blowpipe and Tube among the Yagya Indians of the Peruvian Amazon » in Laura M. Rival and Neil L. Whitehead, eds, Beyond the Visible and the Material, Oxford University Press, New York, pp. 81-89.

2002 "Armados hasta los dientes. Los trofeos de dientes humanos en Amazonía " in Thomas Myers and María Cipolletti, eds, Artifacts and Society in Amazonia, Bonner Amerikanistische Studen, 36, Bonn, pp. 115126. 
Clastres Pierre

1977 "Archéologie de la violence : la guerre dans les sociétés primitives », Libre, 1, pp. 137-173.

Descola Philippe

1986a "Contrôle social de la transgression et guerre de vendetta dans le haut amazone ", Droit et cultures, 11, pp. 137-140.

1986b La Nature domestique : symbolisme et praxis dans l'écologie des Achuar, Éditions de la Maison des sciences de l'homme, Paris.

1993a Les Lances du crépuscule. Relations Jivaros, Haute-Amazonie, Plon, Paris.

1993b «Les affinités sélectives. Alliance, guerre et prédation dans l'ensemble Jivaro », L'Homme, 126-128, avr.-déc., pp. 171-190.

2001 Leçon inaugurale faite le jeudi 29 mars au Collège de France, Chaire d'Anthropologie de la Nature, Collège de France, Paris.

Descola Philippe et Michel Izard

1991 " Guerre » in Pierre Bonte et Michel Izard, éds, Dictionnaire de l'ethnologie et de l'anthropologie, PUF, Paris, pp. 313-316.

D'ONOFrio Salvatore

à paraître La parenté ayoré.

Escobar Ticio

1993 La belleza de los otros. Arte indigena del Paraguay, RPediciones, Asunción.

Fausto Carlos

2000 "Of enemies and pets : warfare and shamanism in Amazonia », American Ethnologist, 26, pp. 933-956.

2001 Inimigos fiéis. Historia, guerre e xamanismo na Amazônia, Edusp, Sao Paulo.

Fischermann Bernd

1976 «Los Ayoreode » in J. Riester, ed., En busca de la Loma Santa, Amigos del Libro, La Paz-Cochabamba.

1988 Zur Weltsicht der Ayoréode Ostboliviens, Rheinische Friedrich-WilhelmsUniversität, Bonn.

HARRIS Marvin

1984 "A Cultural Materialist Theory of Band and Village Warfare: the Yanoamo Test » in B. Ferguson, ed., Warfare, Culture, and Environment, Academic Press, New York, pp. 111-140.

Héritier Françoise, éd.

1996 De la violence I, Odile Jacob, Paris.

1999 De la violence II, Odile Jacob, Paris.

IDOYaGa Molina Anatilde

1989 «Significaciones de la mítica de susmaningai (el coraje) en la cosmovisión ayoreo $"$, Mitológicas, pp. 31-37.

LÉvi-Strauss Claude

1943 « Guerre et commerce chez les Indiens de l'Amérique du Sud », Renaissance, I (1-2), janv.-juin, pp. 122-139.

1985 La Potière jalouse, Plon, Paris. 
2000a "Apologues des amibes » in Jean-Luc Jamard, Emmanuel Terray et Margharita Xanthakou, éds, En substances. Textes pour Françoise Héritier, Fayard, Paris, pp. 493-496.

2000b "Gli uomini visti da un'ameba », La Repubblica, 9 marzo.

Mauro Tullio de

1970 "Introduzione » in Corso di linguistica generale, Laterza, Bari, pp. v-xxxIx.

Mashnshnek Olga Celia

1990 «Las nociones míticas en la economía de producción de los ayoreo del Chaco boreal ", Scripta Ethnologica-Supplementos, CAEA, Buenos Aires, pp. 119-139.

Menget Patrick

2000 "Togore, le bras-trophée », Os índios, 9, Museu Nacional de Etnologia, Lisboa, pp. 117-119.

New Tribes Mission

2000 Ayoré-English Dictionary (appendixes : English-Ayoré Dictionary), New Tribes Mission, Stanford, 2 vol.

OLRIK Axel

1973 «La costruzione del racconto : le leggi epiche », Uomo \& cultura, VI (11-12), pp. 197-232.

ONG Walter J.

1982 Orality and Literacy. The Technologizing of the Word, Routledge, London.

Overing Joanna

1993 «Death and the Loss of Civilized Predation among the Piaroa of the Orinoco Basin ", L'Homme, 33 (2-4), pp. 191-211.

Pagés Larraya Fernando

1973 "El complejo cultural de la locura en los moros-ayoreo », Acta psiquiátrica psicológica de América Latina, 19, pp. 253-264.

Pallestrini Luciana y José A. Perasso

1988 Jeguakáva. Arte plumario indigena del Paraguay, Editorial El Lector, Asunción.

SAHAGÚn Bernardino de (Fr.)

1982 Historia general de las cosas de Nueva España, Editorial Porrúa, México.

SCHMEdA-Hirschmann Guillermo

1994 «Etnobotánica ayoreo », Revista Universum, pp. 107-156.

Sebag Lucien

1965 «Le chamanisme Ayoréo », L'Homme, V (1), janvier-mars, pp. 7-32 ; (2), avril-juin, pp. 92-122.

SHERzER Joel

1982 "Poetic Structuring of Kuna Discours : the Line ", Language and Society, 2, pp. 371-390.

1987 "A Discourse-Centered Approach to Language and Culture ", American Anthropologist, 89, pp. 295-309. 
SusNik Branka J.

1963 "La lengua de los ayoweos-moros. Nociones generales », Boletín de la Sociedad Cientifica del Paraguay y del Museo Etnográfico, Etnolingüística-8, Asunción.

1990 "Guerra Transito Subsistencia (Ambito americano)», Manuales del Museo Etnográfico " Andrés Barbero », Asunción.

TAYLOR Anne-Christine

1985 "L'art de la réduction : la guerre et les mécanismes de la différenciation tribale dans la culture Jivaro », Journal de la Société des Américanistes, 71, pp. 159-173.

1994 «Les complexités de l'alliance dans les rituels de chasse aux têtes des Shuar (Jivaro) de l'Équateur » in Françoise Héritier et Elisabeth Copet-Rougier, éds, Les Complexités de l'alliance, IV, Archives contemporaines, Paris, pp. 73-105, coll. «Économie, politique et fondements symboliques de l'alliance ".

URBAN Greg

1990 A Discourse-Centered Approach to Culture. Native South American Myths and Rituals, University of Texas Press, Austin.

Viveiros de CASTRo Eduardo

1992 From the Enemy's Point of View : Humanity and Divinity in an Amazonian Society, Chicago University Press, Chicago.

1993 "Alguns aspectos da afinidade no dravidianato Amazônico » in Eduardo Viveiros de Castro y Manuela Carneiro da Cunha, eds, Amazônia : Etnología e história indigena, Fundaçao de Amparo à Pesquisa do Estado de São Paulo, São Paulo, pp. 150-210.

WiLbert Johannes and Karin Simoneau

1988 Folk Literature of the Ayoreo Indians, UCLA Latin American Center Publications, Los Angeles.

\section{ZANARDINI Giuseppe}

1994 Beyori ga yicatecarori. Relatos ayoreo-español, Editorial Don Bosco, Asunción. 